\title{
Signal-to-noise ratio of the bispectral analysis of speckle interferometry
}

\author{
Tadashi Nakajima \\ Division of Physics, Mathematics, and Astronomy, California Institute of Technology, Pasadena, California 91125
}

Received February 8, 1988; accepted April 26, 1988

\begin{abstract}
Monte Carlo simulations of an atmospheric phase screen, based on a Kolmogorov spectrum of phase fluctuations, were performed. Speckle patterns produced from the phase screens were used to derive statistical properties of power spectra and bispectra of speckle interferograms. We present the bispectral modulation transfer function and its signal-to-noise ratio at high light levels. The results confirm the validity of a heuristic treatment based on an interferometric picture of speckle pattern formation in deriving the attenuation factor and the signal-to-noise ratio of the bispectral modulation transfer function in the mid-spatial-frequency range. The derived modulation transfer function is also interpreted in terms of the signal-to-noise ratio at low light levels. A general expression of the signal-to-noise ratio of the bispectrum is derived as a function of the transfer functions of the telescope, the number of speckles, and the mean photon counts in the mid-spatial-frequency range.
\end{abstract}

\section{INTRODUCTION}

Speckle interferometry ${ }^{1}$ was first extended to full imaging with phase by Knox and Thompson. ${ }^{2}$ A more powerful imaging technique, based the use of closure phase, ${ }^{3}$ was developed in radio astronomy. Independently, for optical wavelengths, a method to extract closure-phase information from speckle observations by means of the bispectrum was developed by the Erlangen group. ${ }^{4}$ So far, the method, called bispectral analysis, has been successful in recovering a 10th-magnitude multiple stellar system. ${ }^{5}$ However, the potential and the limitations of the method have not yet been investigated fully both in sensitivity and resolution. It is important to quantify the behavior of the signal-to-noise ratio (SNR) of the bispectrum, which depends on both the spatial frequency and the light level.

The analysis of the SNR of the bispectral analysis is parallel to that of the power spectrum analysis and comprises two stages. First the modulation transfer functions (MTF's) that describe the combined effect of the telescope and the atmospheric disturbance are obtained by treating an incoming light as a wave and treating a speckle interferogram as an intensity distribution. By taking the influence of photon noise into account, the SNR of the bispectrum at arbitrary light levels is determined as a function of the classical MTF's and the mean photon counts.

In most discussions of the SNR in the literature on speckle interferometry, MTF's are derived in the mid-spatial-frequency range, based on the heuristic interferometric view (HIV) of the image-forming process. ${ }^{6}$ From this point of view, a speckle pattern is regarded as a random interference pattern produced by a partially coherent incident wave. The validity of this heuristic treatment is known empirically in the case of the power-spectrum analysis. The effect of the atmospheric disturbance is included in only one parameter, the coherence length. The existence of the steep Kolmogorov spectrum in phase fluctuations suggests that there may be some important effects that are not predicted by this simple approach. A more thorough derivation of the power- spectrum MTF, based on the phase structure function of the Kolmogorov theory, was derived by Korff, ${ }^{7}$ who used a semianalytical approach that took the atmospheric turbulence properly into account. The derivation of the power-spectrum MTF is close to the limit of what can be done analytically. In order to obtain higher-order MTF's, such as the bispectral MTF, that take the Kolmogorov theory into account, it is necessary to resort to Monte Carlo simulations. This method enables us to test the predictions of the HIV of the bispectral analysis, ${ }^{8-10}$ and it is shown here that those predictions can be used as a guide to the correct first-order results. Predictions of the HIV are summarized in Appen$\operatorname{dix}$ A of this paper.

The modeling of the photodetection process, based on the rules of conditional statistics, and its application to the power-spectrum analysis were given by Goodman and Belsher, ${ }^{11-13}$ who formulated an unbiased estimator of the classical power spectrum from an ensemble of photon-noiselimited images. They also obtained an expression for the SNR of the power spectrum in terms of the classical MTF and the mean photon counts. Their analysis is applicable to non-photon-counting detection. In other words, they treated a case in which average photon counts per image were measurable but neither the positions of the individual photons nor the total photon counts of individual images were known. Dainty and Greenaway ${ }^{14}$ applied the approach of Goodman and Belsher ${ }^{11-13}$ to photon-counting detection and pointed out that an unbiased estimator of the power spectrum is given in the same manner as in the case of nonphoton-counting detection but the expression for the SNR is different. Since the photon-noise bias can be removed in each frame, the variance of the power spectrum does not include terms originating from the fluctuations of the bias.

Wirnitzer ${ }^{15}$ gave an unbiased estimator of the classical bispectrum, applying the method of Goodman and Belsher ${ }^{11-13}$ to the bispectral analysis for photon-counting detection. Wirnitzer also obtained the SNR in the highand low-light limits by evaluating the corresponding leading terms in the power of photon counts. This was the first 
realistic attempt to estimate the limiting magnitude of the bispectral analysis. However, as was first pointed out by Karbelkar and Nityananda, ${ }^{9}$ the classical bispectral MTF adopted by Wirnitzer and the SNR in the high-light limit do not agree with those derived from the treatment based on the HIV.

Monte Carlo simulations of an atmospheric phase screen, based on the Kolmogorov spectrum derived by Tatarskii, 16,17 were made in order to study statistical properties of the bispectral MTF at high light levels. The algorithm and the computation are described in Sections 2 and 3, respectively. The results of the simulations for a $2-\mathrm{m}$ telescope are presented in Section 4. The results are compared with those obtained by using the interferometric view in Section 5 . The MTF obtained by the simulations is reinterpreted to yield the SNR at low light levels in Section 6 . In Section 7 the discussion of the SNR is generalized to arbitrary light levels and arbitrary telescope sizes by modeling of the photodetection process and the approximate MTF's in the midfrequency range. Finally, in Section 8 the SNR in the recovered map is considered. An estimate of the practical limiting magnitude is discussed along with the limitation in resolution.

\section{ALGORITHM}

The simulations are based on the Kolmogorov theory of turbulence and refractive-index fluctuation ${ }^{16-18}$ and on recent observations of the altitude dependence of the refractive-index structure constant $C_{n}^{2}$ (e.g., the La Silla Seeing Campaign ${ }^{19,20}$ ). The Kolmogorov theory provides mathematical expressions of the atmospheric disturbance on a light-wave propagation as two-dimensional spectral densities of phase fluctuation and amplitude fluctuation and the cross spectral density of the two. On the other hand, recent observations indicate that most of the turbulence is produced at the boundary layer and that the high-altitude turbulence contributes a relatively small fraction of the overall seeing degradation. We therefore assume that the major disturbance appears as phase fluctuations in the near-field limit and that the amplitude fluctuation (scintillation) and the cross correlation of the phase and the amplitude fluctuations are negligible to first order.

In the near-field limit, the spectral density of the phase fluctuation at the aperture plane of a telescope is given by

$$
F_{S}\left(\kappa_{r}\right)=0.0332 \pi k^{2}\left[\int C_{n}{ }^{2}(L) \mathrm{d} L\right] \kappa_{r}^{-11 / 3},
$$

where $\kappa_{r}=\left(\kappa_{x}^{2}+\kappa_{y}^{2}\right)^{1 / 2}$ is the two-dimensional radial spatial frequency, $k$ is the wave number, and $\int C_{n}{ }^{2}(L) \mathrm{d} L$ is the integrated structure constant of the refractive-index fluctuation over the optical path through a turbulent medium.

Since the spectral density constrains the frequency-dependent variance of the fluctuation but not the probability distribution, we further assume a Gaussian probability distribution with zero mean. In what follows, $\Phi(\mathbf{x})$ denotes the phase at the aperture plane of a telescope and is a real function of $\mathbf{x}$, and $\tilde{\Phi}(\kappa)$ denotes its Fourier transform, which is a conjugate-symmetric-complex function of $\kappa$. At each point over one half of the $\kappa$ space, $\tilde{\Phi}(\kappa)$, a complex random number whose modulus is a Gaussian random number with a variance of $F_{S}\left(\kappa_{r}\right) \Delta \kappa$ and whose phase is a uniform random number betwéen 0 and $2 \pi$, is generated. $\Delta \kappa$ denotes an area in $\kappa$ space equaling $\left(2 \pi / l_{\max }\right)^{2}$, where $l_{\max }$ is the size of the square phase screen. A conjugate-symmetric-to-real Fourier transform from $x$ to $\mathbf{x}$ space then creates a monochromatic phase screen $\Phi(\mathbf{x})$ at $\lambda=2 \pi / k$.

An idealized telescope is simulated simply by a circular aperture on the phase screen. At each point of the aperture plane, the complex amplitude $\Psi(\mathbf{x})=\exp [i \Phi(\mathbf{x})]$ is calculated. Another Fourier transform simulates the light-wave propagation from the aperture plane to the image plane, and from the squared modulus of the Fourier transform of $\Psi(\mathbf{x})$, a monochromatic speckle pattern, $|\tilde{\Psi}(\mathbf{s})|^{2}$, is obtained, where $\mathbf{s}$ denotes the coordinate on the image plane. Since the phase fluctuations are simply proportional to the wave number $k$, the finite-bandwidth effect is taken into account by averaging over monochromatic speckle patterns at equally spaced wave numbers covering the bandpass. Thus, from one evaluation of $\Psi(\mathbf{x})$, multiple $|\tilde{\Psi}(\mathbf{s})|^{2}$ are generated and averaged to produce one speckle interferogram $I(\mathbf{s})$. If Taylor's hypothesis of frozen-in turbulence ${ }^{21}$ and a uniform translation by a constant wind velocity are assumed, a continuous observation can be simulated by considering a series of apertures displaced by a distance that is typically the coherence length, $r_{c}$.

In the data reduction, many short-exposure frames are processed to derived statistics. The Fourier transform $\tilde{I}_{j}(\mathbf{u})$ of the speckle interferogram of the $j$ th frame $I_{j}(\mathbf{s})$ is taken to form the bispectrum,

$$
\tilde{B}_{j}^{(3)}\left(\mathbf{u}_{1}, \mathbf{u}_{2}\right)=\tilde{I}_{j}\left(\mathbf{u}_{1}\right) \tilde{I}_{j}\left(\mathbf{u}_{2}\right) \tilde{I}_{j}\left(-\mathbf{u}_{1}-\mathbf{u}_{2}\right)
$$

where $\mathbf{u}$ denotes the spatial frequency on the image plane. For $\dot{n}$ frames, both the sum of bispectra,

$$
\sum_{j=1}^{n} \tilde{B}_{j}^{(3)}\left(\mathbf{u}_{1}, \mathbf{u}_{2}\right)
$$

and the sum of square moduli,

$$
\sum_{j=1}^{n}\left|\tilde{B}_{j}^{(3)}\left(\mathbf{u}_{1}, \mathbf{u}_{2}\right)\right|^{2}
$$

are calculated. The unbiased estimator of the ensembleaverage bispectrum is

$$
\left\langle\tilde{B}^{(3)}\left(\mathbf{u}_{1}, \mathbf{u}_{2}\right)\right\rangle=\frac{\sum_{j=1}^{n} \tilde{B}_{j}^{(3)}\left(\mathbf{u}_{1}, \mathbf{u}_{2}\right)}{n},
$$

where \langle\rangle indicates an ensemble average. Likewise the unbiased estimator of the variance of the bispectrum per frame is

$$
\sigma^{2}\left[\tilde{B}^{3}\left(\mathbf{u}_{1}, \mathbf{u}_{2}\right)\right]=\frac{\sum_{j=1}^{n}\left|\tilde{B}_{j}^{(3)}\left(\mathbf{u}_{1}, \mathbf{u}_{2}\right)\right|^{2}-n\left|\left\langle\tilde{B}^{3}\left(\mathbf{u}_{1}, \mathbf{u}_{2}\right)\right\rangle\right|^{2}}{n-1} .
$$

In the case of the bispectral MTF, the mean value is real, ${ }^{3}$ since the atmospheric disturbance is statistically isotropic and the ideal telescope is static and symmetric. After aver- 
aging over enough samples, the SNR of the MTF per frame is defined as

$$
\operatorname{SNR}\left[\tilde{B}^{(3)}\left(\mathbf{u}_{1}, \mathbf{u}_{2}\right)\right]=\frac{\operatorname{Re}\left[\left\langle\tilde{B}^{(3)}\left(\mathbf{u}_{1}, \mathbf{u}_{2}\right)\right\rangle\right]}{\left\{\sigma^{2}\left[\tilde{B}^{(3)}\left(\mathbf{u}_{1}, \mathbf{u}_{2}\right)\right]\right\}^{1 / 2}} .
$$

Henceforth a SNR is taken to mean a SNR per frame unless specified otherwise. The SNR in the recovered map is discussed in Section 8.

\section{COMPUTATION}

The simulations were made at a wavelength $\lambda=0.55 \mu \mathrm{m}$ with a fractional bandwidth of 0.1 . At this wavelength, the integrated structure constant of the refractive-index fluctuation,

$$
\int C_{n}{ }^{2}(L) \mathrm{d} L=5 \times 10^{-13} \mathrm{~m}^{1 / 3},
$$

was adopted corresponding to 1 -arcsec seeing. This is approximately Roddier's value. ${ }^{22}$ It was found experimentally that five monochromatic speckle interferograms produced at equally spaced wave numbers within the bandpass were enough to obtain a reasonable averaged speckle interferogram.

In order to include wave-front degradations at small scales, the sampling interval on the phase screen must be significantly smaller than the coherence length $r_{c}$. On the other hand, the linear size of the phase screen $\left(l_{\max }\right)$ must be significantly larger than the primary mirror of the telescope so that the large-scale disturbance is simulated properly. Both the sampling interval and the ratio between the size of the phase screen and the diameter of the primary mirror $l_{\max } / D$ were determined empirically. The sampling interval $\Delta l$ was chosen to be $2 \mathrm{~cm}$, so that 49 phase data were obtained within a square area of $r_{c}^{2}$ for $r_{c}=14 \mathrm{~cm}$. It was found that fluctuations with correlation scales larger than four times the telescope diameter mainly caused image wandering but did not affect the power spectrum of the bispectrum of a speckle interferogram. The ratio $l_{\max } / D$ must be at least 4 .

The maximum practical array size on the computer used, a $\mathrm{VAX} / 750$, is $512^{2}$ when the memory access time and the CPU

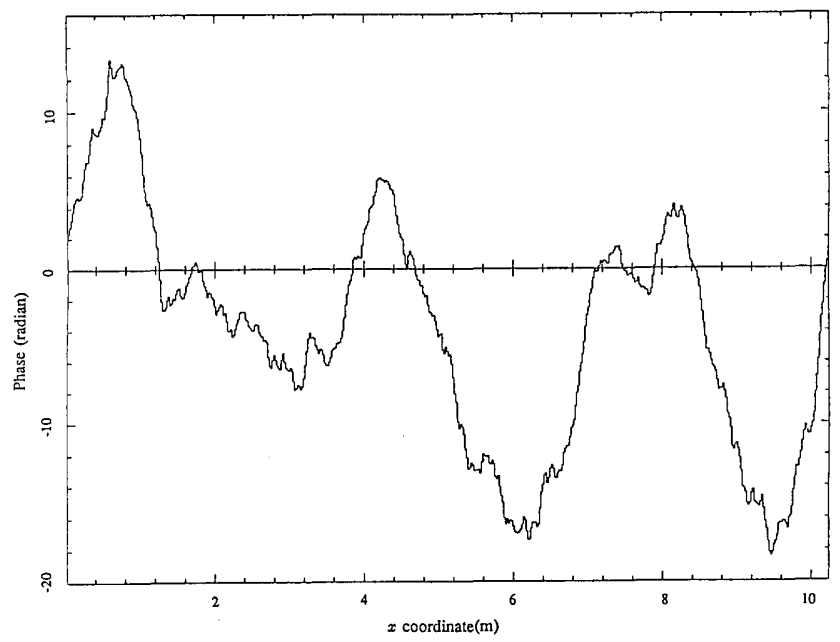

Fig. 1. Cross section of a simulated phase screen, plotted as a function of the $x$ coordinate on the aperture plane.

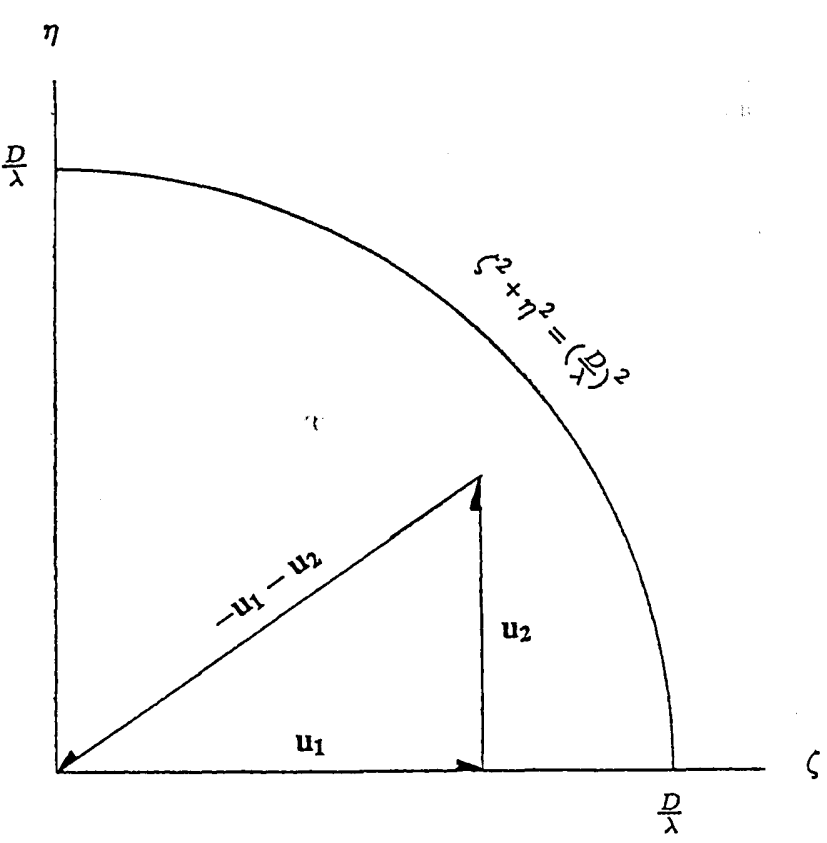

Fig. 2. Two-dimensional coordinate system $(\zeta, \eta)$, adopted to represent a two-dimensional cross section of the four-dimensional bispectrum $\tilde{b}^{(3)}(\zeta, 0,0, \eta)$, and that of the SNR. $\mathbf{u}_{1}=(\zeta, 0)$ and $\mathbf{u}_{2}=(0$, $\eta)$ are perpendicular to each other, and the third spatial frequency $-\mathbf{u}_{1}-\mathbf{u}_{2}=(-\zeta,-\eta)$ has the largest modulus $\left(\zeta^{2}+\eta^{2}\right)^{1 / 2}$. Therefore the circle $\zeta^{2}+\eta^{2}=(D / \lambda)^{2}$ forms the boundary, where $D / \lambda$ is the telescope-cutoff frequency.

time for the fast Fourier transform are taken into account. When the array size is combined with the sampling interval $\Delta l$, the size of phase screen is $(512 \Delta l)^{2} \approx 10 \mathrm{~m} \times 10 \mathrm{~m}$. Since the size of the phase screen is still smaller than the typical outer scale of the atmospheric turbulence, the Kolmogorov theory can safely be assumed. The maximum primary mirror size of the resultant simulated telescope is about $2.5 \mathrm{~m}$ or 128 pixels in diameter. In practice, we concentrated on a 2 $\mathrm{m}$ telescope but also made calculations for a 1-m telescope for comparison. In producing images, $256^{2}$-sized fast Fourier transforms were made to satisfy the Nyquist sampling requirement. A cross section of a phase screen is shown in Fig. 1.

Unfortunately the entire four-dimensional bispectrum is beyond the capacity of the computer used. It is, however, possible to get a good insight into the MTF from a twodimensional cross section of the bispectrum, since the atmospheric disturbance was already assumed to be locally isotropic in the Kolmogorov theory. The cross section of the bispectral MTF was chosen so that $\mathbf{u}_{1}$ and $\mathbf{u}_{2}$ are perpendicular to each other because of the convenience in drawing two-dimensional contour maps (Fig. 2). Therefore in a fourdimensional expression the cross section is

$$
\tilde{B}^{(3)}(\zeta, 0,0, \eta)=\tilde{I}(\zeta, 0) \tilde{I}(0, \eta) \tilde{I}(-\zeta,-\eta) .
$$

It should be noted that the third spatial frequency $\left(-u_{1}-\right.$ $\mathbf{u}_{2}$ ) has the largest modulus, $\left(\zeta^{2}+\eta^{2}\right)^{1 / 2}$, among the three and that the circle,

$$
\zeta^{2}+\eta^{2}=(D / \lambda)^{2}
$$

forms the boundary of the bispectrum, where $D / \lambda$ is the telescope cutoff frequency. 


\section{RESULTS OF THE SIMULATIONS}

The results of the computations are the normalized bispectral MTF's, defined as

$$
\left\langle\tilde{b}^{(3)}\left(\mathbf{u}_{1}, \mathbf{u}_{2}\right)\right\rangle=\frac{\left\langle\tilde{B}^{(3)}\left(\mathbf{u}_{1}, \mathbf{u}_{2}\right)\right\rangle}{\left\langle\tilde{B}^{(3)}(0,0)\right\rangle} .
$$

Since fluctuations in the total intensity $\tilde{I}(0)=\int I(\mathbf{x}) \mathrm{d} \mathbf{x}$ are not considered,

$$
\left\langle\tilde{B}^{(3)}(0,0)\right\rangle=\tilde{I}(0)^{3}=\text { const., }
$$

and then

$$
\left\langle\tilde{b}^{(3)}\left(\mathbf{u}_{1}, \mathbf{u}_{2}\right)\right\rangle=\left\langle\tilde{i}\left(\mathbf{u}_{1}\right) \tilde{i}\left(\mathbf{u}_{2}\right) \tilde{i}\left(-\mathbf{u}_{1}-\mathbf{u}_{2}\right)\right\rangle,
$$

where $\tilde{i}(\mathbf{u})=\tilde{I}(\mathbf{u}) / \tilde{I}(0)$. For the same reason, the SNR in the normalized bispectral MTF is

$$
\operatorname{SNR}\left[\tilde{b}^{(3)}\left(\mathbf{u}_{1}, \mathbf{u}_{2}\right)\right]=\operatorname{SNR}\left[\tilde{B}^{(3)}\left(\mathbf{u}_{1}, \mathbf{u}_{2}\right)\right] .
$$

The $\zeta$ and $\eta$ axes on the $\zeta-\eta$ plane correspond to the normalized power-spectrum MTF:

$$
\begin{aligned}
\left\langle\tilde{b}^{(3)}(\zeta, 0,0,0)\right\rangle & =\langle\tilde{i}(\zeta, 0) \tilde{i}(0,0) \tilde{i}(-\zeta, 0)\rangle \\
& =\left\langle\tilde{i}(\zeta, 0) 1 \tilde{i}(\zeta, 0)^{*}\right\rangle \\
& =\left\langle|\tilde{i}(\zeta, 0)|^{2}\right\rangle,
\end{aligned}
$$

and, similarly,

$$
\left\langle\tilde{b}^{(3)}(0,0,0, \eta)\right\rangle=\left\langle|\tilde{i}(0, \eta)|^{2}\right\rangle .
$$

The normalized MTF of a 2-m telescope is shown in Fig. 3. The statistics are derived from 500 interferograms sampled from independent portions of 50 different phase screens to ensure the statistical independence of instantaneous bispectra. One pixel in spatial frequency corresponds to 0.173

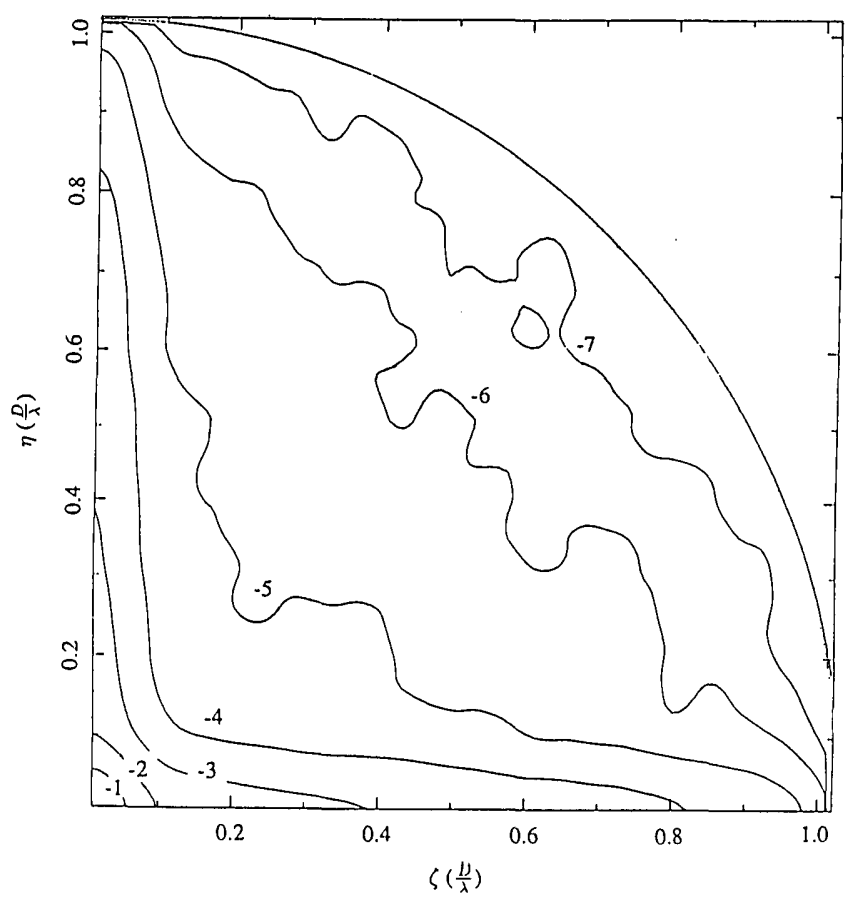

Fig. 3. Contour map of the normalized bispectral $\operatorname{MTF} \tilde{b}^{(3)}(\zeta, 0,0$, $\eta$ ) for a 2-m telescope, drawn on the $\zeta-\eta$ plane in a logarithmic scale. The numbers labeling contours indicate powers of 10 .

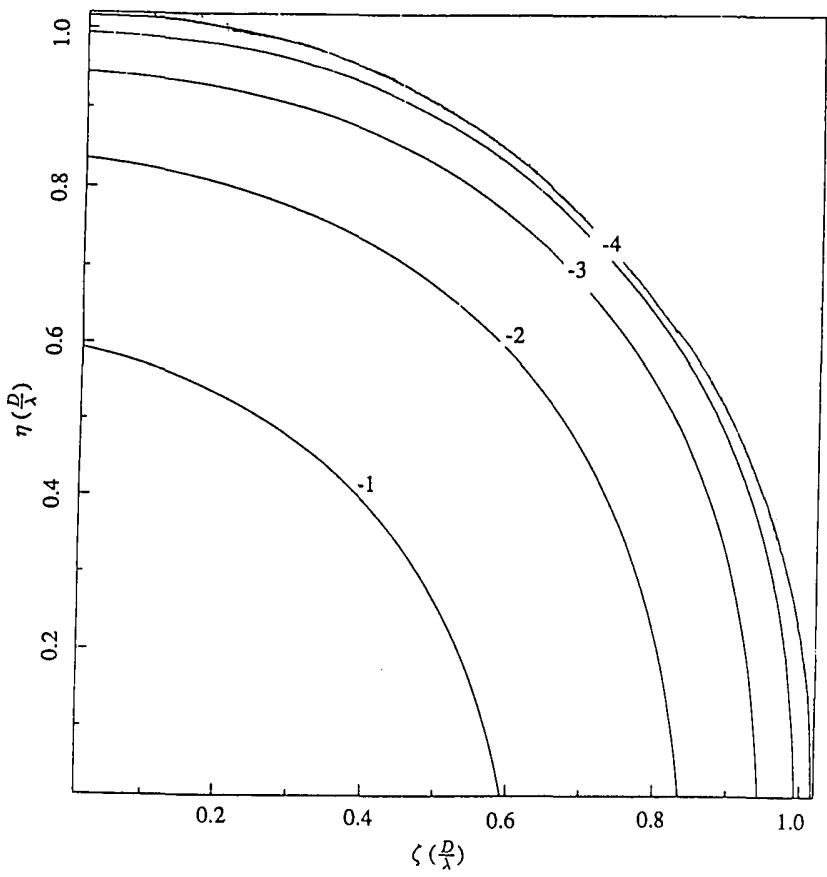

Fig. 4. Contour map of the normalized OTF of the telescope, $\tilde{t}^{(3)}(\zeta$, $0,0, \eta)$, drawn on the $\zeta-\eta$ plane in a logarithmic scale. The numbers labeling contours indicate powers of 10 .

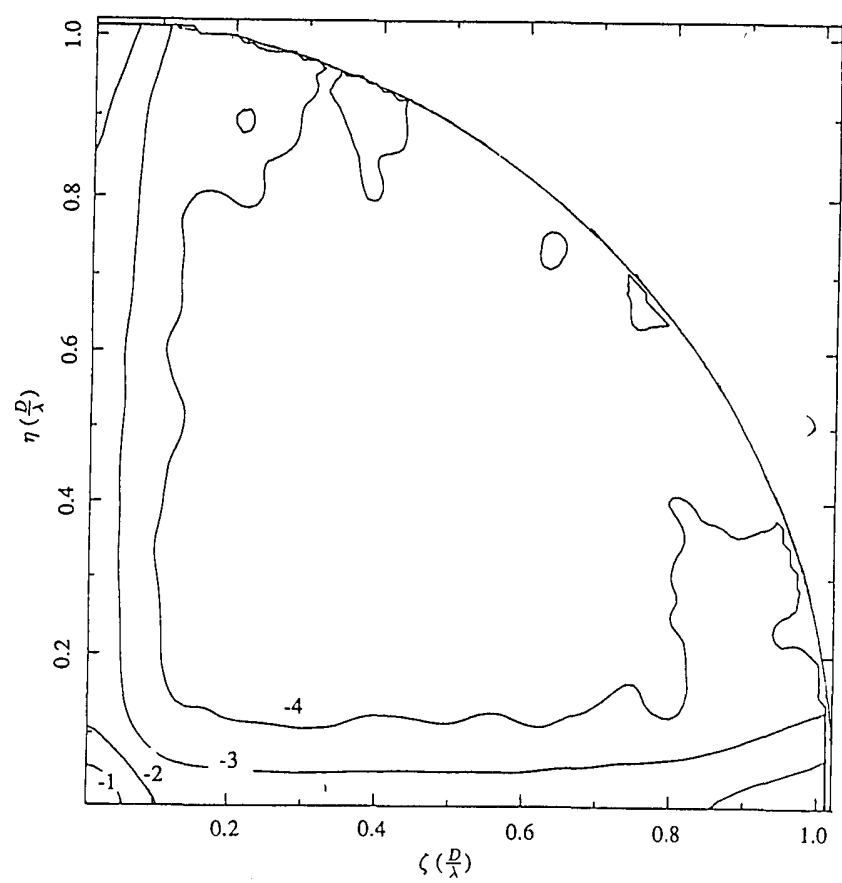

Fig. 5. Contour map of the attenuation factor, $\tilde{a}^{(3)}(\zeta, 0,0, \eta)$ for a 2 $\mathrm{m}$ telescope, drawn on the $\zeta-\eta$ plane in a logarithmic scale. The numbers labeling contours indicate powers of 10 .

$\operatorname{arcsec}^{-1}=(1.95 \mathrm{~cm}) /(0.55 \mu \mathrm{m})=0.01 D / \lambda$, and the $102 \mathrm{nd}$ pixel corresponds to the telescope cutoff frequency, $D / \lambda$.

In understanding the physics of the results, it is convenient to introduce a concept of the attenuation of the bispectral MTF, since the atmospheric disturbance is regarded as a low-pass filter of the spatial-frequency information. The attenuation factor $(\mathrm{ATF})$ of the bispectral $\mathrm{MTF}, \tilde{a}^{(3)}(\zeta, 0,0$, 
$\eta$ ) is defined along with the optical transfer function (OTF) of the telescope, $\tilde{t}^{(3)}(\zeta, 0,0, \eta)$, as

$$
\left\langle\tilde{a}^{(3)}(\zeta, 0,0, \eta)\right\rangle=\frac{\left\langle\tilde{b}^{(3)}(\zeta, 0,0, \eta)\right\rangle}{\tilde{t}^{(3)}(\zeta, 0,0, \eta)}
$$

where $\tilde{t}^{(3)}(\zeta, 0,0, \eta)$ is static and real. The OTF is the normalized bispectrum of the Airy pattern and thus is the normalized MTF under the coherent illumination. It could also be interpreted as the relative weight of the frequency components or the relative redundancy of the triangular baselines (both closed and nonclosed) on the primary mirror. To avoid confusion, it should be noted that the attenuation is the combined effect of the atmosphere and the optics and thus that the ATF depends on the OTF even for a given atmospheric condition. The OTF and the ATF are shown in Figs. 4 and 5, respectively. The OTF is a monotonically decreasing function of $\zeta$ and $\eta$. It is fairly flat at $\left(\zeta^{2}+\eta^{2}\right)^{1 / 2}$ $\leq 0.5 \mathrm{D} / \lambda$ and then falls more steeply at higher frequencies.

The ATF, and thus the MTF, behave in a more complicated manner. The SNR of the classical MTF or the saturated SNR at high light levels is shown in Fig. 6. The behavior of the SNR is quite similar to that of the ATF. Semiquantitatively, the contour maps of the ATF and the SNR can be classified into five distinct regions in spatial frequency as follows.

\section{A. Low-Frequency Region $\left[\left(\zeta^{2}+\eta^{2}\right]^{1 / 2} \leq 0.1 D / \lambda\right]$}

The ATF is larger than 0.01 and the SNR is larger than unity. The information of this region originates from the envelopes of instantaneous interferograms. Even this lowfrequency region has better information than a seeing disk obtained by a long time exposure, since the effect of image wandering is removed.

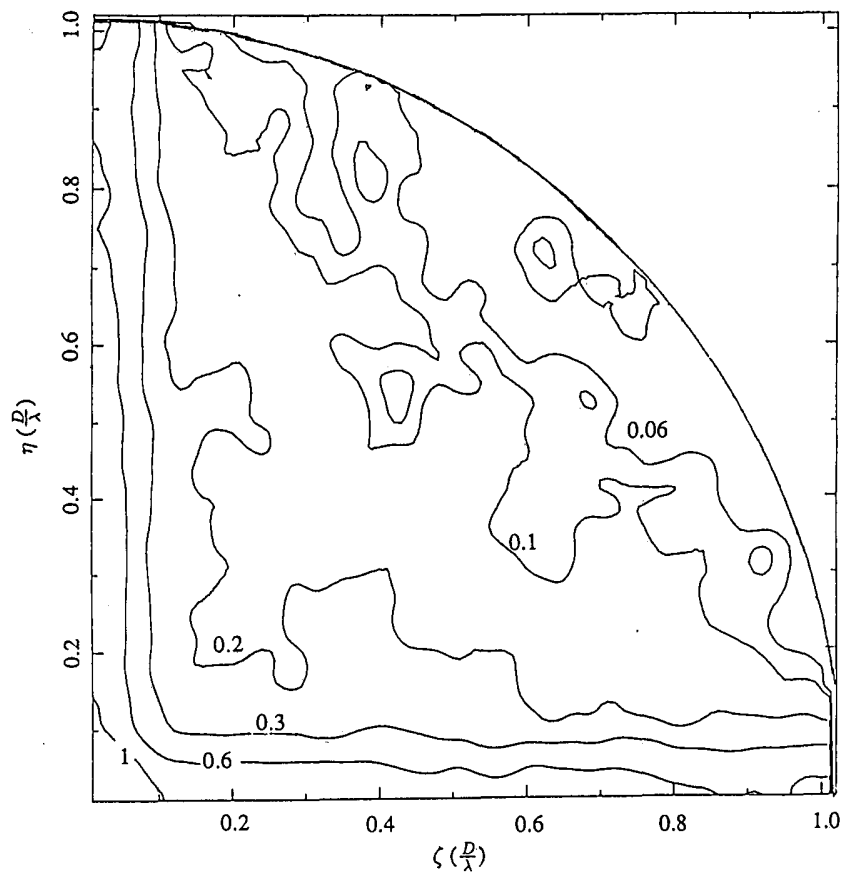

Fig. 6. Contour map of the SNR of the bispectral MTF $\operatorname{SNR}\left[\tilde{b}^{(3)}(\zeta\right.$, $0,0, \eta)]$ for a single frame obtained with a $2-\mathrm{m}$ telescope, drawn on the $\zeta-\eta$ plane. This map shows the saturated SNR at high light levels.

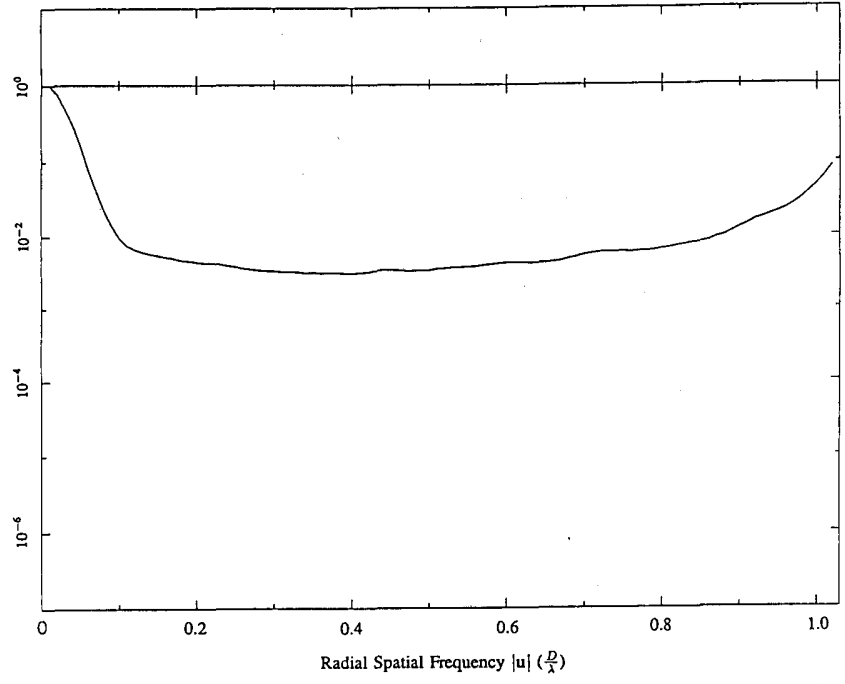

Fig. 7. Power-spectrum atmospheric transfer function $|\tilde{a}(\mathbf{u})|^{2}$, plotted as a function of the modulus of the radial spatial frequency $|\mathbf{u}|$

\section{B. On-Axis Region $(\zeta=\mathbf{0}, \eta=\mathbf{0})$}

The power-spectrum ATF is plotted as a function of radial frequency in Fig. 7, since it is statistically isotropic. The ATF falls off steeply at low frequencies $(\leq 0.1 D / \lambda)$ and then levels between $0.1 \mathrm{D} / \lambda$ and $0.8 \mathrm{D} / \lambda$ at around $4 \times 10^{-3}$. Above $0.8 D / \lambda$, the ATF slowly rises up to $10^{-1}$ at $D / \lambda$. This increase in the ATF at high spatial frequencies is not fast enough to compensate for the steep fall of the OTF, and the power-spectrum MTF monotonically falls off, as is shown on the axes in Fig. 3. The SNR is larger than 0.8 up to $D / \lambda$.

\section{Near-Axis Region $(\zeta \leq 0.1 D / \lambda$, or $\eta \leq 0.1 D / \lambda)$}

The ATF falls off steeply to $10^{-4}$, and the SNR decreases to 0.3 as the plot moves vertically away from each axis. The bispectral components are the combination of the low-frequency Fourier components and the power spectrum. Phases of the bispectral components in this region are effectively local phase differences of nearby Fourier components, which are used in the Knox-Thompson method. ${ }^{2}$

\section{Mid-Frequency Region $\left[\zeta, \eta \geq 0.1 D / \lambda\right.$ and $\left(\zeta^{2}+\eta^{2}\right)^{1 / 2}$ $\leq 0.8 D / \lambda]$}

A large triangular plateau of the ATF with a mean value of 3 $\times 10^{-5}$ is evident in Fig. 5. In this region, the behavior of the MTF is determined mainly by that of the OTF. The MTF falls from $10^{-4}$ to $10^{-6}$. The SNR is between 0.1 and 0.2 .

\section{E. High-Frequency Region $\left[\zeta^{2}+\eta^{2} \approx(D / \lambda)^{2}\right]$}

The diffraction-limited information lies in this region. Because of the steep fall of the OTF, the MTF is very small $\left(\leq 10^{-7}\right)$. The SNR is smaller than 0.1.

\section{COMPARISON WITH THE HEURISTIC INTERFEROMETRIC VIEW}

It is interesting to compare the results described in section four with the predictions obtained by using the HIV of the image-forming process. A brief description of this view is given in Appendix $\mathrm{A}$, and a detailed discussion is found in 
Ref. 10. The HIV predicts that the power-spectrum ATF is approximated by $n_{s}^{-1}=\left(r_{\mathrm{c}} / D\right)^{2}$, where $n_{s}$ is the number of speckles, and that the SNR of the power spectrum is unity in the mid-frequency range. It also estimates that the bispectral ATF in the mid-frequency range is about $n_{s}{ }^{-2}=\left(r_{c} / D\right)^{4}$ and that the SNR is given by $n_{s}^{-1 / 2}=r_{c} / D$. The bispectral $\mathrm{ATF}$ and its SNR are therefore related by

$$
\left\langle\tilde{a}^{(3)}\left(\mathbf{u}_{1}, \mathbf{u}_{2}\right)\right\rangle \approx\left\{\operatorname{SNR}\left[\tilde{b}^{(3)}\left(\mathbf{u}_{1}, \mathbf{u}_{2}\right)\right]\right\}^{4} .
$$

Thus the similarity of the contour maps of the ATF and the SNR, which are plotted in logarithmic scale in Figs. 5 and 6 , is naturally explained by the HIV. For $r_{c}=14 \mathrm{~cm}$ and $D=2$ $\mathrm{m}, n_{s}=204$. The flat portion of the simulated powerspectrum ATF between $0.2 \mathrm{D} / \lambda$ and $0.8 \mathrm{D} / \lambda$ is $4 \times 10^{-3}$ on average, whereas the value predicted by the HIV is $5 \times 10^{-3}$. The SNR of the power spectrum lies between 0.6 and 0.8 and is approximately unity. At the mid-frequency region, the simulated bispectral ATF has an average value of $3 \times 10^{-5}$, whereas the value predicted by using the HIV is $2 \times 10^{-5}$. We consider this agreement good.

The simulations were also made for a 1-m-diameter telescope. For a telescope of this size the midfrequency of the power spectrum ranges from $0.3 \mathrm{D} / \lambda$ to $0.6 \mathrm{D} / \lambda$, and the simulated power-spectrum ATF is $3 \times 10^{-2}$, whereas the predicted value is $2 \times 10^{-2}$. The SNR of the power spectrum is between 0.7 and 1.0. The simulated bispectral ATF at the mid frequency is $2 \times 10^{-3}$ in average, which is somewhat larger than the predicted value, $4 \times 10^{-4}$. The agreement is not so good as for a 2-m telescope.

The rise of the power-spectrum ATF at the high-frequency region ( $\geq 0.8 D / \lambda$ for $D=2 \mathrm{~m}$ ) can be interpreted qualitatively by the HIV. At the high-frequency region, the redundancy (or OTF) of the baselines is so small and the identical baselines are so localized on the primary mirror that the phasors of those baselines are correlated and increase the ATF. The approximate validity of the HIV of the imageforming process is confirmed by the simulations. The simulations also clarified the boundaries of the mid frequency for given apertures. Because of the higher redundancy and the wider mid-frequency range, the predictions made by using the HIV work better for larger telescopes.

\section{SIGNAL-TO-NOISE RATIO AT LOW LIGHT LEVELS}

At low light levels, the SNR per frame of an unbiased estimator of the classical bispectral MTF is approximated by

$$
\left\langle\tilde{b}^{(3)}\left(\mathbf{u}_{1}, \mathbf{u}_{2}\right)\right\rangle \times \bar{N}^{3 / 2},
$$

where $\bar{N}$ is the average photon count per frame. ${ }^{15}$ Therefore the contour map of $\left\langle\tilde{b}^{(3)}\left(\mathbf{u}_{1}, \mathbf{u}_{2}\right)\right\rangle$ can be converted immediately to that of the SNR.

Figure 8 shows the SNR of a $V=12.3$ magnitude star with a 2-m telescope after integrating $10^{4}$ frames; this magnitude corresponds to one photon per speckle in a $10 \%$ fractional bandwidth, with $10 \%$ efficiency of the observing system and a 10 -msec integration time. The $\mathrm{SNR}=3$ contour reaches the diffraction limit on the axes but stays near the axis as $\zeta$ or $\eta$ increases. The slope of the contours is the steepest diagonally. Figure 9 shows SNR $=3$ contours according to the brightness of the sources. At 9.0 magnitudes the bispectral analysis is diffraction limited in the sense of a $3 \sigma$ detection,

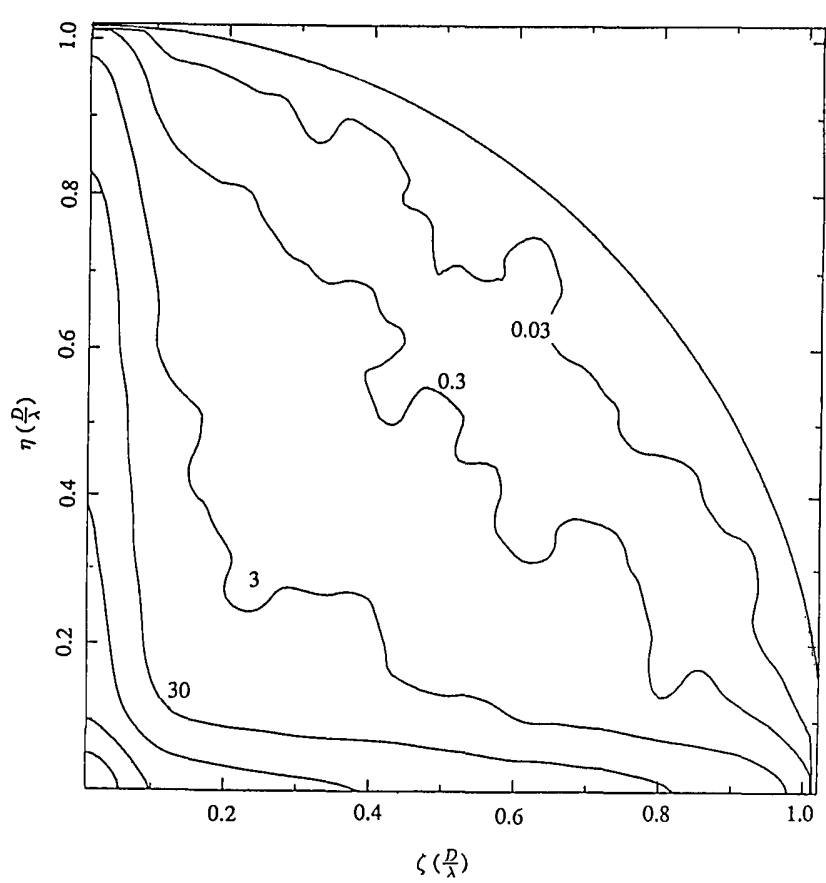

Fig. 8. Contour map of the SNR for a $V=12.3$ magnitude star obtained with a $2-\mathrm{m}$ telescope after integrating $10^{4}$ frames, assuming a $10 \%$ observing efficiency, a $10 \%$ fractional bandwidth and a $10-$ msec integration time. These brightness and observing efficiencies correspond to 1 photon per speckle.

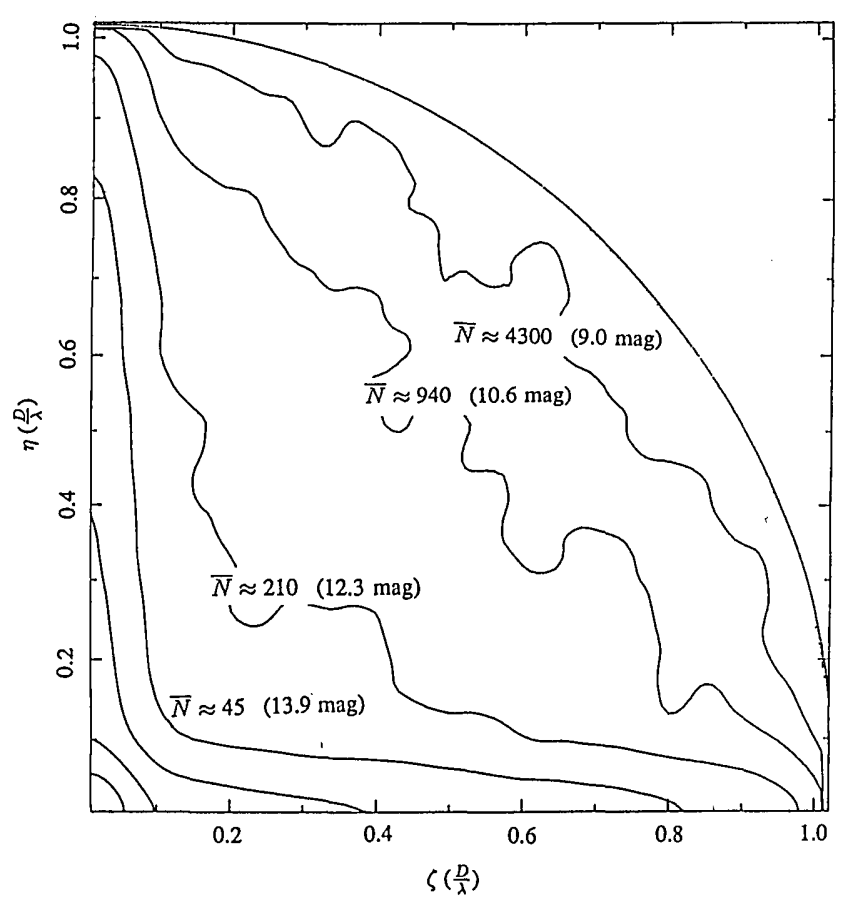

Fig. 9. Behavior of $3 \sigma$ contours, plotted according to the light levels. Magnitudes are calculated for the same conditions as for Fig. 8.

whereas at 13.9 magnitudes even the power-spectrum analysis is not necessarily diffraction limited, and the region of high SNR is strictly near the axes.

The contour maps immediately show that the power spectrum in general has a better SNR than the bispectrum. The closure-phase information obtained by near-axis bispectral 
components is effectively the local phase differences of neighboring Fourier components. For a simple source such as a multiple stellar system, the autocorrelation function contains most of the source structure. The behavior of the phase in Fourier space is fairly regular, and thus the local phase differences are enough to recover a full image. Hofmann and Weigelt ${ }^{5}$ used only the $5 \%$ of the bispectrum near the axes with the highest SNR for their image recovery. The result of the simulations is consistent with their observations. The wide mid-frequency range contains global closure-phase information with lower quality. For a complicated source, mid-frequency components may be crucial in recovering a full image. Intensive computations are required for utilization of the full bispectrum.

\section{SIGNAL-TO-NOISE RATIO AT ARBITRARY LIGHT LEVELS}

In the simulations, the incoming light is treated as a wave. Thus, from the point of view of photon detection, a limiting case with an infinite number of photons is considered. In this section, by using the modeling of the photodetection process by Goodman and Belsher ${ }^{11-13,23}$ and following the treatment of the influence of photon noise on the bispectral analysis by Wirnitzer, ${ }^{15}$ the derivation of an unbiased estimator of the classical bispectrum is reviewed, and then an expression for the SNR of the bispectral MTF is obtained as a function of the mean photon count, the OTF's of telescopes, and the number of speckles.

We consider a speckle observation by using a photoncounting detector that records the positions of individual photons detected on the image plane. The raw intensity of the $j$ th frame is given as

$$
D_{j}(\mathbf{x})=\sum_{k=1}^{N_{j}} \delta\left(\mathbf{x}-\mathbf{x}_{k}\right),
$$

where $x_{k}$ is the position of the $k$ th photon and $N_{j}$ is the total number of photons. The Fourier transform of Eq. (7.1) is

$$
\begin{aligned}
\tilde{D}_{j}(\mathbf{u}) & =\int \sum_{k=1}^{N_{j}} \delta\left(\mathbf{x}-\mathbf{x}_{k}\right) \exp (i \mathbf{u x}) \mathrm{d} \mathbf{x} \\
& =\sum_{k=1}^{N_{j}} \exp \left(i \mathbf{u} \mathbf{x}_{k}\right) .
\end{aligned}
$$

The bispectrum of the raw data is given as

$$
\begin{aligned}
\tilde{D}_{j}^{(3)}\left(\mathbf{u}_{1}, \mathbf{u}_{2}\right) & =\tilde{D}_{j}\left(\mathbf{u}_{1}\right) \tilde{D}_{j}\left(\mathbf{u}_{2}\right) \tilde{D}_{j}\left(-\mathbf{u}_{1}-\mathbf{u}_{2}\right) \\
& =\sum_{k=1}^{N_{j}} \sum_{l=1}^{N_{j}} \sum_{m=1}^{N_{j}} \exp \left\{i\left[\mathbf{u}_{1}\left(\mathbf{x}_{k}-\mathbf{x}_{m}\right)+\mathbf{u}_{2}\left(\mathbf{x}_{l}-\mathbf{x}_{m}\right)\right]\right\} .
\end{aligned}
$$

The expected value of $\tilde{D}_{j}^{(3)}\left(\mathbf{u}_{1}, \mathbf{u}_{2}\right)$ is evaluated over the conditional statistics of $\mathbf{x}_{k} s, N_{j}$, and the rate function $\lambda_{j}(\mathbf{x})$, which is proportional to the classical intensity, $I_{j}(\mathbf{x})$. For a given $N_{j}$ and $\lambda_{j}(\mathbf{x})$, the event locations $\mathbf{x}$ are independent random variables with a common probability-density function,

$$
p_{j}(\mathbf{x})=\frac{\lambda_{j}(\mathbf{x})}{\int \lambda_{j}(\mathbf{x}) \mathrm{d} \mathbf{x}}=\frac{I_{j}(\mathbf{x})}{\int I_{j}(\mathbf{x}) \mathrm{d} \mathbf{x}} .
$$

The characteristic function of $p_{j}(\mathbf{x})$ equals the normalized Fourier transform of the classical intensity distribution $\tilde{i}_{j}(\mathbf{u})$ :

$$
\begin{aligned}
\tilde{p}_{j}(\mathbf{u}) & =\int p_{j}(\mathbf{x}) \exp (i \mathbf{u x}) \mathrm{d} \mathbf{x} \\
& =\frac{\int I_{j}(\mathbf{x}) \exp (i \mathbf{u x}) \mathrm{d} \mathbf{x}}{\int I_{j}(\mathbf{x}) \mathrm{d} \mathbf{x}} \\
& =\frac{\tilde{I}_{j}(\mathbf{u})}{\tilde{I}_{j}(\mathbf{0})} \\
& =\tilde{i}_{j}(\mathbf{u}) .
\end{aligned}
$$

$\tilde{D}_{j}{ }^{(3)}\left(\mathbf{u}_{1}, \mathbf{u}_{2}\right)$ is averaged first over the conditional statistics of $\mathbf{x}_{k}, \mathbf{x}_{l}$, and $\mathbf{x}_{m}$ and then over $N_{j}$ and $\lambda_{j}(\mathbf{x})$. The starting point is the evaluation of

$$
\begin{aligned}
E_{k l m}\left[\tilde{D}_{j}^{(3)}\left(\mathbf{u}_{1}, \mathbf{u}_{2}\right)\right] & \\
= & E_{k l m}\left(\sum_{k=1}^{N_{j}} \sum_{l=1}^{N_{j}} \sum_{m=1}^{N_{j}} \exp \left\{i\left[\mathbf{u}_{1}\left(\mathbf{x}_{k}-\mathbf{x}_{m}\right)+\mathbf{u}_{2}\left(\mathbf{x}_{l}-\mathbf{x}_{m}\right)\right]\right\}\right) \\
= & \sum_{k=1}^{N_{j}} \sum_{l=1}^{N_{j}} \sum_{m=1}^{N_{j}} E_{k l m}\left(\exp \left\{i\left[\mathbf{u}_{1}\left(\mathbf{x}_{k}-\mathbf{x}_{\dot{m}}\right)+\mathbf{u}_{2}\left(\mathbf{x}_{l}-\mathbf{x}_{m}\right)\right]\right\}\right),
\end{aligned}
$$

where $E_{k l m}$ stands for an average over $\mathbf{x}_{k}, \mathbf{x}_{l}$, and $\mathbf{x}_{m}$. The $N_{j}^{3}$ terms are classified as follows:

(1) For $k=l=m$ and $N_{j}$ terms,

$$
E_{k l m}(1)=\int p_{j}\left(\mathbf{x}_{k}\right) \mathrm{d} \mathbf{x}_{k}=1
$$

(2) For $k \neq l=m$ and $N_{j}\left(N_{j}-1\right)$ terms,

$$
\begin{aligned}
& E_{k l m}\left\{\exp \left[i \mathbf{u}_{1}\left(\mathbf{x}_{k}-\mathbf{k}_{l}\right)\right]\right\} \\
& \quad=\iint \exp \left[i \mathbf{u}_{1}\left(\mathbf{x}_{k}-\mathbf{x}_{l}\right)\right] p_{j}\left(\dot{\mathbf{x}}_{k}\right) p_{j}\left(\mathbf{x}_{l}\right) \mathrm{d} \mathbf{x}_{k} \mathrm{~d} \mathbf{x}_{l} \\
& \quad=\left[\int p_{j}\left(\mathbf{x}_{k}\right) \exp \left(i \mathbf{u}_{1} \mathbf{x}_{k}\right) \mathrm{d} \mathbf{x}_{k}\right]\left[\int p_{j}\left(\mathbf{x}_{l}\right) \exp \left(-i \mathbf{u}_{1} \mathbf{x}_{l}\right) \mathrm{d} \mathbf{x}_{l}\right] \\
& \quad=\tilde{i}_{j}\left(\mathbf{u}_{1}\right) \tilde{i}_{j}\left(-\mathbf{u}_{1}\right) \\
& \quad=\left|\tilde{i}_{j}\left(\mathbf{u}_{1}\right)\right|^{2},
\end{aligned}
$$

where $\left|\tilde{i}_{j}\left(\mathbf{u}_{1}\right)\right|^{2}$ is the normalized power spectrum.

(3) For $k=m \neq l$ and $N_{j}\left(N_{j}-1\right)$ terms,

$$
E_{k l m}\left\{\exp \left[i \mathbf{u}_{1}\left(\mathbf{x}_{l}-\mathbf{x}_{m}\right)\right]\right\}=\left|\tilde{i}_{j}\left(\mathbf{u}_{2}\right)\right|^{2} .
$$

(4) For $k=l \neq m$ and $N_{j}\left(N_{j}-1\right)$ terms,

$$
E_{k l m}\left\{\exp \left[i\left(-\mathbf{u}_{1}-\mathbf{u}_{2}\right)\left(\mathbf{x}_{l}-\mathbf{x}_{m}\right)\right]\right\}=\left|\tilde{i}_{j}\left(-\mathbf{u}_{1}-\mathbf{u}_{2}\right)\right|^{2} .
$$


(5) For $k \neq l \neq m$ and $N_{j}\left(N_{j}-1\right)\left(N_{j}-2\right)$ terms,

$$
\begin{aligned}
& E_{k l m}\left(\exp \left\{i\left[\mathbf{u}_{1}\left(\mathbf{x}_{k}-\mathbf{x}_{m}\right)+\mathbf{u}_{2}\left(\mathbf{x}_{l}-\mathbf{x}_{m}\right)\right]\right\}\right) \\
&= {\left[\int p_{j}\left(\mathbf{x}_{k}\right) \exp \left(i \mathbf{u}_{1} \mathbf{x}_{k}\right) \mathrm{d} \mathbf{x}_{k}\right]\left[\int p_{j}\left(\mathbf{x}_{l}\right) \exp \left(i \mathbf{u}_{2} \mathbf{x}_{l}\right) \mathrm{d} \mathbf{x}_{l}\right] } \\
& \times\left(\int p_{j}\left(\mathbf{x}_{m}\right) \exp \left[i\left(-\mathbf{u}_{1}-\mathbf{u}_{2}\right) \mathbf{x}_{m}\right] \mathrm{d} \mathbf{x}_{m}\right) \\
&= \tilde{i}_{j}\left(\mathbf{u}_{1}\right) \tilde{i}_{j}\left(\mathbf{u}_{2}\right) \tilde{i}_{j}\left(-\mathbf{u}_{1}-\mathbf{u}_{2}\right) \\
&= \tilde{b}_{j}^{(3)}\left(\mathbf{u}_{1}, \mathbf{u}_{2}\right),
\end{aligned}
$$

where $\tilde{b}_{j}^{(3)}\left(\mathbf{u}_{1}, \mathbf{u}_{2}\right)$ is the normalized bispectrum.

Thus the average of $\tilde{D}_{j}^{(3)}\left(\mathbf{u}_{1}, \mathbf{u}_{2}\right)$ over the statistics of $\mathbf{x}_{k}, \mathbf{x}_{l}$, and $\mathbf{x}_{m}$ is

$$
\begin{aligned}
E_{k l m}\left[\tilde{D}_{j}^{(3)}\left(\mathbf{u}_{1}, \mathbf{u}_{2}\right)\right] & \\
= & N_{j}+N_{j}\left(N_{j}-1\right)\left[\left|\tilde{i}_{j}\left(\mathbf{u}_{1}\right)\right|^{2}+\left|\tilde{i}_{j}\left(\mathbf{u}_{2}\right)\right|^{2}+\left|\tilde{i}_{j}\left(-\mathbf{u}_{1}-\mathbf{u}_{2}\right)\right|^{2}\right] \\
& +N_{j}\left(N_{j}-1\right)\left(N_{j}-2\right) \times \tilde{b}_{j}^{(3)}\left(\mathbf{u}_{1}, \mathbf{u}_{2}\right) .
\end{aligned}
$$

Next $E_{k l m}\left[\tilde{D}_{j}{ }^{(3)}\left(\mathbf{u}_{1}, \mathbf{u}_{2}\right)\right]$ is averaged over the Poisson statistics of $N_{j}$. For Poisson statistics,

$$
E\left[N_{j}\left(N_{j}-1\right) \ldots\left(N_{j}-r+1\right)\right]=\bar{N}_{j}^{r},
$$

where $\bar{N}_{j}$ denotes the Poisson mean of $N_{j}$. For a given rate function $\lambda_{j}(\mathbf{x})$,

$$
\begin{aligned}
E_{k l m, N_{j}}\left[\tilde{D}_{j}^{(3)}\left(\mathbf{u}_{1}, \mathbf{u}_{2}\right)\right] & \\
= & \bar{N}_{j}+\bar{N}_{j}^{2}\left[\left|\tilde{i}_{j}\left(\mathbf{u}_{1}\right)\right|^{2}+\left|\tilde{i}_{j}\left(\mathbf{u}_{2}\right)\right|^{2}+\left|\tilde{i}_{j}\left(-\mathbf{u}_{1}-\mathbf{u}_{2}\right)\right|^{2}\right] \\
& +\bar{N}_{j}{ }^{3} \tilde{b}_{j}^{(3)}\left(\mathbf{u}_{1}, \mathbf{u}_{2}\right) .
\end{aligned}
$$

Finally, averaging over the ensemble of $\lambda_{j}(\mathbf{x})$ or $p_{j}(\mathbf{x})$, yields $E\left[\tilde{D}^{(3)}\left(\mathbf{u}_{1}, \mathbf{u}_{2}\right)\right]$

$$
\begin{aligned}
= & \bar{N}_{j}+\bar{N}_{j}^{2}\left[\left\langle\left|\tilde{i}\left(\mathbf{u}_{1}\right)\right|^{2}\right\rangle+\left\langle\left|\tilde{i}\left(\mathbf{u}_{2}\right)\right|^{2}\right\rangle+\left\langle\left|\tilde{i}\left(-\mathbf{u}_{1}-\mathbf{u}_{2}\right)\right|^{2}\right\rangle\right] \\
& +\bar{N}_{j}^{3}\left\langle\tilde{b}^{(3)}\left(\mathbf{u}_{1}, \mathbf{u}_{2}\right)\right\rangle .
\end{aligned}
$$

If $\bar{N}_{j}$ does not fluctuate from frame to frame, i.e.,

$$
E\left(\bar{N}_{j}^{r}\right)=E\left(N_{j}\right)^{r}=\bar{N}^{r},
$$

then, for an arbitrary $r$,

$$
\begin{aligned}
E\left[\tilde{D}^{(3)}\left(\mathbf{u}_{1}, \mathbf{u}_{2}\right)\right]= & \bar{N}+\bar{N}^{2}\left[\left\langle\left|\tilde{i}\left(\mathbf{u}_{1}\right)\right|^{2}\right\rangle+\left\langle\left|\tilde{i}\left(\mathbf{u}_{2}\right)\right|^{2}\right\rangle\right. \\
& \left.+\left\langle\left|\tilde{i}\left(-\mathbf{u}_{1}-\mathbf{u}_{2}\right)\right|^{2}\right\rangle\right]+\bar{N}^{3}\left\langle\tilde{b}^{(3)}\left(\mathbf{u}_{1}, \mathbf{u}_{2}\right)\right\rangle .
\end{aligned}
$$

In order to express an unbiased estimator of $\left\langle\tilde{b}^{(3)}\left(\mathbf{u}_{1}, \mathbf{u}_{2}\right)\right\rangle$ by using the quantities observed directly,

$$
E\left[|\tilde{D}(\mathbf{u})|^{2}\right]=\bar{N}+\bar{N}^{2}\left\langle|\tilde{i}(\mathbf{u})|^{2}\right\rangle
$$

is useful. This equation was first obtained by Goodman and Belsher for non-photon-counting detection and was also derived by Dainty and Greenaway ${ }^{14}$ for photon-counting detection. From it we obtain

$$
\begin{aligned}
\bar{N}^{3}\left\langle\tilde{b}^{(3)}\left(\mathbf{u}_{1}, \mathbf{u}_{2}\right)\right\rangle= & E\left\{\tilde{D}^{(3)}\left(\mathbf{u}_{1}, \mathbf{u}_{2}\right)-\left[\left|\tilde{D}\left(\mathbf{u}_{1}\right)\right|^{2}\right.\right. \\
& \left.\left.+\left|\tilde{D}\left(\mathbf{u}_{2}\right)\right|^{2}+\left|\tilde{D}\left(-\mathbf{u}_{1}-\mathbf{u}_{2}\right)\right|^{2}-2 \bar{N}\right]\right\} .
\end{aligned}
$$

Thus an unbiased estimator of the bispectrum for the $j$ th frame becomes

$$
\begin{aligned}
\tilde{Q}_{j}^{(3)}\left(\mathbf{u}_{1}, \mathbf{u}_{2}\right)= & \tilde{D}_{j}^{(3)}\left(\mathbf{u}_{1}, \mathbf{u}_{2}\right)-\left[\left|\tilde{D}_{j}\left(\mathbf{u}_{1}\right)\right|^{2}\right. \\
& \left.+\left|\tilde{D}_{j}\left(\mathbf{u}_{2}\right)\right|^{2}+\left|\tilde{D}_{j}\left(-\mathbf{u}_{1}-\mathbf{u}_{2}\right)\right|^{2}-2 \bar{N}_{j}\right],
\end{aligned}
$$

where the terms in square brackets represent the photonnoise bias. Equation (7.20) was first obtained by Wirnitzer. ${ }^{15}$ This estimator can be rewritten as

$$
\tilde{Q}_{j}^{(3)}\left(\mathbf{u}_{1}, \mathbf{u}_{2}\right)=\sum_{k \neq l \neq m} \exp \left\{i\left[\mathbf{u}_{1}\left(\mathbf{x}_{k}-\mathbf{x}_{m}\right)+\mathbf{u}_{2}\left(\mathbf{x}_{l}-\mathbf{x}_{m}\right)\right]\right\} .
$$

Since the observables are the positions of individual photons, it is also possible to calculate the $N_{j}\left(N_{j}-1\right)\left(N_{j}-2\right)$ exponential terms directly through Eq. (7.21). The absolute minimum number of photons per frame is 3 , since triple cross correlations of different photon events contribute to the unbiased estimator of the classical bispectrum.

The next goal is to find the variance of the unbiased estimator $\widetilde{Q}_{j}^{(3)}\left(\mathbf{u}_{1}, \mathbf{u}_{2}\right)$ and its SNR per frame. In evaluating the variance $\sigma^{2}\left[\tilde{Q}_{j}{ }^{(3)}\left(\mathbf{u}_{1}, \mathbf{u}_{2}\right)\right]$, it is necessary to calculate $E\left[\left|\tilde{Q}_{j}{ }^{(3)}\left(\mathbf{u}_{1}, \mathbf{u}_{2}\right)\right|^{2}\right]$. The derivation is systematic but lengthy and is given in Appendix B. Here only the resultant expression is presented:

$$
\begin{aligned}
& \sigma^{2}\left[\tilde{Q}_{j}^{(3)}\left(\mathbf{u}_{1}, \mathbf{u}_{2}\right)\right]=\bar{N}^{3}\left[1+\left\langle\left|\tilde{i}\left(\mathbf{u}_{1}-\mathbf{u}_{2}\right)\right|^{2}\right\rangle+\left\langle\left|\tilde{i}\left(2 \mathbf{u}_{1}+\mathbf{u}_{2}\right)\right|^{2}\right\rangle\right. \\
& +\left\langle\left|\tilde{i}\left(\mathbf{u}_{1}+2 \mathbf{u}_{2}\right)\right|^{2}\right\rangle+\left\langle\tilde { b } ^ { ( 3 ) } \left(\mathbf{u}_{1}-\mathbf{u}_{2}, \mathbf{u}_{1}\right.\right. \\
& \left.\left.\left.+2 \mathbf{u}_{2}\right)\right\rangle+\left\langle\tilde{b}^{(3)}\left(2 \mathbf{u}_{1}+\mathbf{u}_{2},-\mathbf{u}_{1}+\mathbf{u}_{2}\right)\right\rangle\right] \\
& +\bar{N}^{4}\left[\left\langle\left|\tilde{i}\left(\mathbf{u}_{1}\right)\right|^{2}\right\rangle+\left\langle\left|\tilde{i}\left(\mathbf{u}_{2}\right)\right|^{2}\right\rangle\right. \\
& +\left\langle\left|\tilde{i}\left(-\mathbf{u}_{1}-\mathbf{u}_{2}\right)\right|^{2}\right\rangle+\left\langle\tilde{b}^{(3)}\left(\mathbf{u}_{1},-\mathbf{u}_{2}\right)\right\rangle+\text { c.c. } \\
& +\left\langle\tilde{b}^{(3)}\left(\mathbf{u}_{1}+\mathbf{u}_{2}, \mathbf{u}_{1}\right)\right\rangle+\text { c.c. } \\
& +\left\langle\tilde{b}^{(3)}\left(\mathbf{u}_{2}, \mathbf{u}_{1}+\mathbf{u}_{2}\right)\right\rangle+\text { c.c. } \\
& +\left\langle\left|\tilde{i}\left(\mathbf{u}_{1}\right)\right|^{2}\left|\tilde{i}\left(\mathbf{u}_{1}+2 \mathbf{u}_{2}\right)\right|^{2}\right\rangle \\
& +\left\langle\left|\tilde{i}\left(\mathbf{u}_{2}\right)\right|^{2}\left|\tilde{i}\left(2 \mathbf{u}_{1}+\mathbf{u}_{2}\right)\right|^{2}\right\rangle \\
& +\left\langle\left|\tilde{i}\left(\mathbf{u}_{1}+\mathbf{u}_{2}\right)\right|{ }^{2}\left|\tilde{i}\left(\mathbf{u}_{1}-\mathbf{u}_{2}\right)\right|^{2}\right\rangle \\
& +\left\langle\tilde{f}^{(4)}\left(\mathbf{u}_{1}-\mathbf{u}_{2}, \mathbf{u}_{1}+2 \mathbf{u}_{2},-\mathbf{u}_{1}-\mathbf{u}_{2}\right)\right\rangle+\text { c.c. } \\
& +\left\langle\tilde{f}^{4)}\left(\mathbf{u}_{1}, \mathbf{u}_{1}+2 \mathbf{u}_{2},-2 \mathbf{u}_{1}-\mathbf{u}_{2}\right)\right\rangle+\text { c.c. } \\
& +\left\langle\tilde{f}^{(4)}\left(2 \mathbf{u}_{1}+\mathbf{u}_{2},-\mathbf{u}_{1}+\mathbf{u}_{2},-\mathbf{u}_{1}-\mathbf{u}_{2}\right)\right\rangle \\
& + \text { c.c. }]+\bar{N}^{5}\left\{\left\langle\left|\tilde{i}\left(\mathbf{u}_{1}\right)\right|^{2}\left|\tilde{i}\left(-\mathbf{u}_{1}-\mathbf{u}_{2}\right)\right|^{2}\right\rangle\right. \\
& +\left\langle\left|\tilde{i}\left(\mathbf{u}_{2}\right)\right|^{2}\left|\tilde{i}\left(-\mathbf{u}_{1}-\mathbf{u}_{2}\right)\right|^{2}\right\rangle+\left\langle\left|\tilde{i}\left(\mathbf{u}_{1}\right)\right|^{2} \mid \tilde{i}\left(\mathbf{u}_{2}\right)^{2}\right\rangle \\
& +\left\langle\left|\tilde{i}\left(\mathbf{u}_{1}\right)\right|^{2}\left[\tilde{b}^{(3)}\left(\mathbf{u}_{2}, \mathbf{u}_{1}+\mathbf{u}_{2}\right)+\text { c.c. }\right]\right\rangle \\
& +\left\langle\left|\tilde{i}\left(\mathbf{u}_{2}\right)\right|^{2}\left[\tilde{b}^{(3)}\left(\mathbf{u}_{1}, \mathbf{u}_{1}+\mathbf{u}_{2}\right)+\text { c.c. }\right]\right\rangle \\
& \left.+\left\langle\left|\tilde{i}\left(-u_{1}-u_{2}\right)\right|^{2}\left[\tilde{b}^{(3)}\left(u_{1},-u_{2}\right)+\text { c.c. }\right]\right\rangle\right\} \\
& +\bar{N}^{6}\left[\left\langle\left|\tilde{b}^{(3)}\left(\mathbf{u}_{1}, \mathbf{u}_{2}\right)\right|^{2}\right\rangle-\left|\left\langle\tilde{b}^{(3)}\left(\mathbf{u}_{1}, \mathbf{u}_{2}\right)\right\rangle\right|^{2}\right],
\end{aligned}
$$




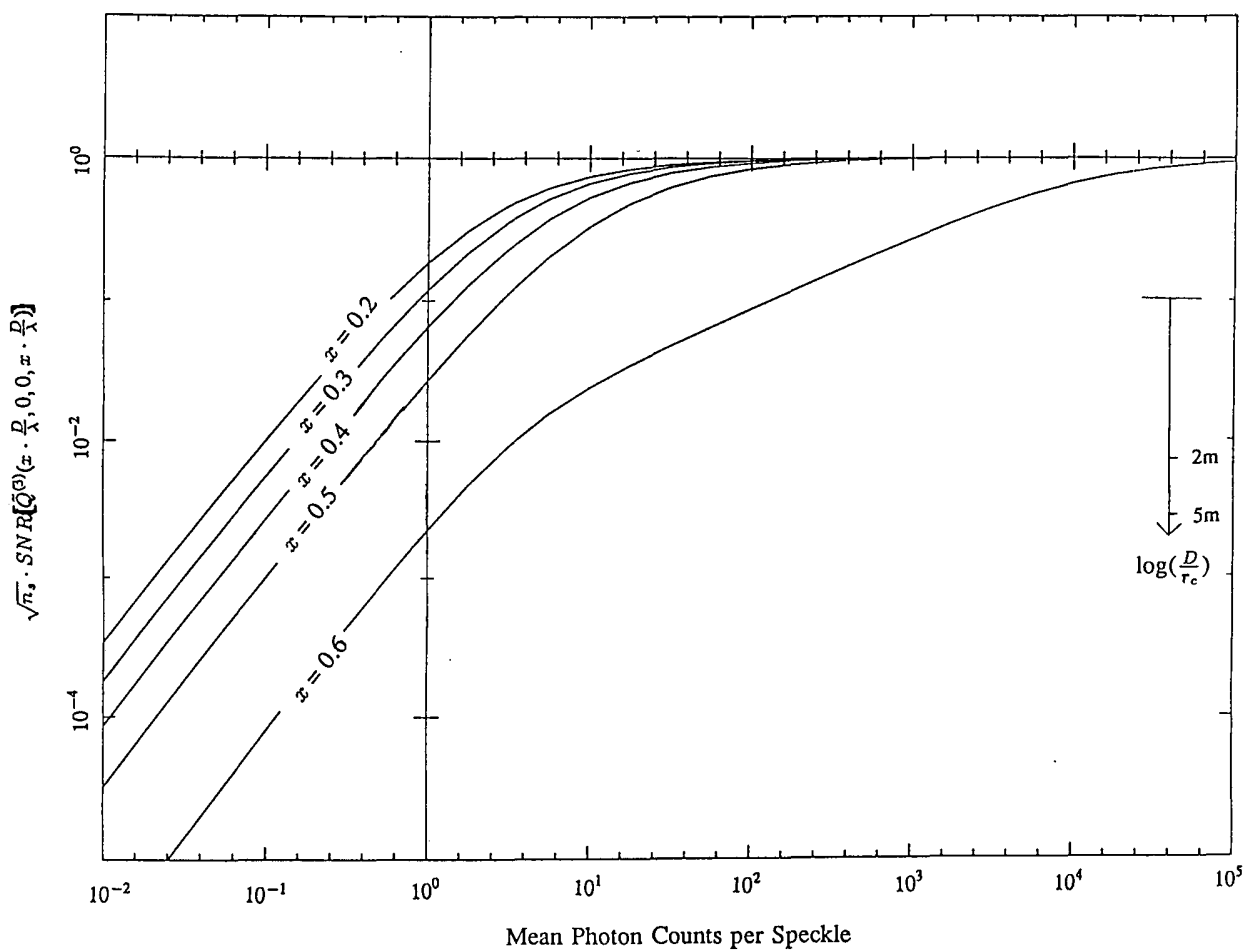

Fig. 10. Light level and spatial-frequency dependence of the $\operatorname{SNR} n_{s}{ }^{1 / 2} \cdot \operatorname{SNR}\left[\tilde{Q}^{(3)}(x \cdot D / \lambda, 0,0, x \cdot D / \lambda)\right]$, which is independent of the size of the telescope, plotted as a function of the mean photon counts per speckle $\bar{n}$ and the normalized spatial frequency $x$. Since $n_{s}{ }^{1 / 2}=D / r_{c}$, the $\mathrm{SNR}$ for a telescope with a diameter of $D$ meters can be obtained by lowering the whole plot by $\log \left(D / r_{c}\right)$, as indicated by the arrow.

$$
\tilde{f}^{(4)}\left(\mathbf{u}_{1}, \mathbf{u}_{2}, \mathbf{u}_{3}\right)=\tilde{i}\left(\mathbf{u}_{1}\right) \tilde{i}\left(\mathbf{u}_{2}\right) \tilde{i}\left(\mathbf{u}_{3}\right) \tilde{i}\left(-\mathbf{u}_{1}-\mathbf{u}_{2}-\mathbf{u}_{3}\right)
$$

is the normalized fourth-order spectrum and c.c. denotes a complex conjugate. The SNR of the unbiased estimator of the bispectrum is given by

$$
\operatorname{SNR}\left[\tilde{Q}^{(3)}\left(\mathbf{u}_{1}, \mathbf{u}_{2}\right)\right]=\frac{\bar{N}^{3}\left\langle\tilde{b}^{(3)}\left(\mathbf{u}_{1}, \mathbf{u}_{2}\right)\right\rangle}{\left\{\sigma^{2}\left[\tilde{Q}^{(3)}\left(\mathbf{u}_{1}, \mathbf{u}_{2}\right)\right]\right\}^{1 / 2}} .
$$

In order to obtain a more useful expression, it is convenient to use approximate attenuation factors of MTF's based on the HIV of the image-forming process (Appendix A). In the mid-frequency range, MTF's are expressed by the number of speckles $n_{s}$ and normalized OTF's of the telescope as follows:

$$
\begin{aligned}
\left\langle|\tilde{i}(\mathbf{u})|^{2}\right\rangle & =n_{s}{ }^{-1}|\tilde{t}(\mathbf{u})|^{2}, \\
\left\langle\tilde{b}^{(3)}\left(\mathbf{u}_{1}, \mathbf{u}_{2}\right)\right\rangle & =n_{s}^{-2} \tilde{t}^{(3)}\left(\mathbf{u}_{1}, \mathbf{u}_{2}\right), \\
\left\langle\tilde{f}^{(4)}\left(\mathbf{u}_{1}, \mathbf{u}_{2}, \mathbf{u}_{3}\right)\right\rangle & =n_{s}^{-3} \tilde{t}^{(4)}\left(\mathbf{u}_{1}, \mathbf{u}_{2}, \mathbf{u}_{3}\right), \\
\left\langle\left|\tilde{i}\left(\mathbf{u}_{1}\right)\right|^{2}\left|\tilde{i}\left(\mathbf{u}_{2}\right)\right|^{2}\right\rangle & =n_{s}{ }^{-2}\left|\tilde{t}\left(\mathbf{u}_{1}\right)\right|{ }^{2}\left|\tilde{t}\left(\mathbf{u}_{2}\right)\right|^{2}, \\
\left\langle\left|\tilde{i}\left(\mathbf{u}_{1}\right)\right|^{2} \tilde{b}^{(3)}\left(\mathbf{u}_{2}, \mathbf{u}_{3}\right)\right\rangle & =n_{s}{ }^{-3}\left|\tilde{t}\left(\mathbf{u}_{1}\right)\right|^{2} \tilde{t}^{(3)}\left(\mathbf{u}_{2}, \mathbf{u}_{3}\right), \\
\left\langle\left|\tilde{b}^{(3)}\left(\mathbf{u}_{1}, \mathbf{u}_{2}\right)\right|^{2}\right\rangle & =n_{s}{ }^{-3}\left|\tilde{t}^{(3)}\left(\mathbf{u}_{1}, \mathbf{u}_{2}\right)\right|^{2}, \\
\left\langle\left.\left\langle\tilde{b}^{(3)}\left(\mathbf{u}_{1}, \mathbf{u}_{2}\right)\right\rangle\right|^{2}\right. & =n_{s}{ }^{-4}\left|\tilde{t}^{(3)}\left(\mathbf{u}_{1}, \mathbf{u}_{2}\right)\right|^{2} .
\end{aligned}
$$

Typically $n_{s}=\left(D / r_{c}\right)^{2} \geq 10^{2}$, and $|\tilde{t}(\mathbf{u})|^{2} \approx 10^{-1}$ in the midfrequency range. By selecting leading terms of each order of $\bar{N}$ in Eq. (7.22), the variance of the unbiased estimator of the classical bispectral MTF, $\sigma^{2}\left[\tilde{Q}^{(3)}\left(\mathbf{u}_{1}, \mathbf{u}_{2}\right)\right]$ is found to be

$$
\begin{aligned}
\sigma^{2}\left[\tilde{Q}^{(3)}\left(\mathbf{u}_{1}, \mathbf{u}_{2}\right)\right]= & \bar{N}^{3}+\bar{N}^{4} n_{s}^{-1} \\
& \times\left[\left|\tilde{t}\left(\mathbf{u}_{1}\right)\right|^{2}+\left|\tilde{t}\left(\mathbf{u}_{2}\right)\right|^{2}+\left|\tilde{t}\left(-\mathbf{u}_{1}-\mathbf{u}_{2}\right)\right|^{2}\right] \\
& +\bar{N}^{5} n_{s}{ }^{-2}\left[\left|\tilde{t}\left(\mathbf{u}_{1}\right)\right|^{2}\left|\tilde{t}\left(-\mathbf{u}_{1}-\mathbf{u}_{2}\right)\right|^{2}\right. \\
& \left.+\left|\tilde{t}\left(\mathbf{u}_{2}\right)\right|^{2}\left|\tilde{t}\left(-\mathbf{u}_{1}-\mathbf{u}_{2}\right)\right|^{2}+\left|\tilde{t}\left(\mathbf{u}_{1}\right)\right|^{2}\left|\tilde{t}\left(\mathbf{u}_{2}\right)\right|^{2}\right] \\
& +\bar{N}^{6} n_{s}{ }^{-3} \tilde{t}^{(3)}\left(\mathbf{u}_{1}, \mathbf{u}_{2}\right) .
\end{aligned}
$$

The SNR of the unbiased estimator is then given by

$$
\operatorname{SNR}\left[\tilde{Q}^{(3)}\left(\mathbf{u}_{1}, \mathbf{u}_{2}\right)\right]=\frac{\bar{N}^{3} n_{s}^{-2} \tilde{t}^{(3)}\left(\mathbf{u}_{1}, \mathbf{u}_{2}\right)}{\left\{\sigma^{2}\left[\tilde{Q}^{(3)}\left(\mathbf{u}_{1}, \mathbf{u}_{2}\right)\right]\right\}^{1 / 2}} .
$$

If the mean number of photons per speckle, $\bar{n}$, is defined as $\bar{n}$ $=\bar{N} / n_{s}$, the SNR can also be expressed as

$$
\operatorname{SNR}\left[\tilde{Q}^{(3)}\left(\mathbf{u}_{1}, \mathbf{u}_{2}\right)\right]=\frac{n_{s}^{-1 / 2} \times \bar{n}^{3 / 2} \times \tilde{t}^{(3)}\left(\mathbf{u}_{1}, \mathbf{u}_{2}\right)}{A^{1 / 2}},
$$

where

$$
\begin{aligned}
A= & 1+\bar{n}\left(\left|\tilde{t}\left(\mathbf{u}_{1}\right)\right|^{2}+\left|\tilde{t}\left(\mathbf{u}_{2}\right)\right|^{2}\right. \\
& \left.+\left|\tilde{t}\left(-\mathbf{u}_{1}-\mathbf{u}_{2}\right)\right|^{2}\right)+\bar{n}^{2}\left(\left|\tilde{t}\left(\mathbf{u}_{1}\right)\right|^{2}\left|\tilde{t}\left(\mathbf{u}_{2}\right)\right|^{2}\right. \\
& +\left|\tilde{t}\left(\mathbf{u}_{1}\right)\right|^{2}\left|\tilde{t}\left(-\mathbf{u}_{1}-\mathbf{u}_{2}\right)\right|^{2}+\left|\tilde{t}\left(\mathbf{u}_{2}\right)\right|^{2} \\
& \left.\times\left|\tilde{t}\left(-\mathbf{u}_{1}-\mathbf{u}_{2}\right)\right|^{2}\right)+\bar{n}^{3}\left|\tilde{t}^{(3)}\left(\mathbf{u}_{1}, \mathbf{u}_{2}\right)\right|^{2}
\end{aligned}
$$

and $n_{s}{ }^{1 / 2} \times \operatorname{SNR}\left[\tilde{Q}^{(3)}\left(\mathbf{u}_{1}, \mathbf{u}_{2}\right)\right]$ is independent of $n_{s}=\left(D / r_{c}\right)^{2}$. In Fig. $10, n_{s}{ }^{1 / 2} \times \operatorname{SNR}\left[\tilde{Q}^{(3)}(x D / \lambda, 0,0, x D / \lambda)\right]$ at $x=(0.2,0.3$, $0.4,0.5,0.6)$ is plotted as a function of $\bar{n}$ in a logarithmic scale. An estimate of the SNR for a telescope with a diameter of $D$ meters can be obtained by lowering the value on the 
plot by $\log \left(D / r_{c}\right)$. It should again be emphasized that the above approximations are valid only in the mid-frequency range.

\section{ESTIMATES OF THE LIMITING MAGNITUDE AND RESOLUTION}

In order to estimate the limiting magnitude, we must first obtain the statistically independent volume of the bispectrum. The HIV suggests that the Fourier components in the mid-frequency range are statistically independent. The volume is proportional to $n_{s}^{2}$, which must be multiplied by a factor related to the symmetry and the boundary of the bispectrum. Wirnitzer estimated the bispectral volume as $n_{s}{ }^{2} / 4$. In Ref. $103 n_{s}{ }^{2} / 4$ was obtained for a square aperture, and $\left(\pi^{2} / 32\right) n_{s}{ }^{2}$ was obtained for a circular aperture, with the assumption of statistical independence of all the baselines. Since only the mid-frequency components are statistically independent, these values give upper limits. An estimate of the SNR of an ideally recovered map from the bispectral $\mathrm{MTF}$ is given as

$$
\operatorname{SNR}(\operatorname{map})=\left(\frac{\pi^{2}}{32} n_{s}{ }^{2}\right)^{1 / 2} \times \overline{\operatorname{SNR}\left[\tilde{Q}^{(3)}\left(\mathbf{u}_{1}, \mathbf{u}_{2}\right)\right]} Z^{1 / 2},
$$

where $\overline{\operatorname{SNR}\left[\widetilde{Q}^{(3)}\left(\mathbf{u}_{1}, \mathbf{u}_{2}\right)\right]}$ is the average SNR over the midfrequency range and $Z$ is the number of frames. As is estimated from Eq. (7.34) and shown in Fig. 10, the SNR at the mid frequency for $\bar{n} \leq 1$ is approximated well by

$$
\operatorname{SNR}\left[\tilde{Q}^{(3)}\left(\mathbf{u}_{1}, \mathbf{u}_{2}\right)\right] \approx n_{s}{ }^{-1 / 2} \bar{n}^{3 / 2} \tilde{t}^{(3)}\left(\mathbf{u}_{1}, \mathbf{u}_{2}\right),
$$

since $A \approx 1$ in (7.34). Equation (8.1) can then be rewritten as

$$
\begin{aligned}
\operatorname{SNR}(\text { map }) & =\left(\frac{\pi^{2}}{32}\right)^{1 / 2}\left(n_{s} \bar{n}^{3}\right)^{1 / 2} \overline{\bar{t}^{(3)}\left(\mathbf{u}_{1}, \mathbf{u}_{2}\right)} \times Z^{1 / 2} \\
& \approx 0.027\left(n_{s} \bar{n}^{3}\right)^{1 / 2} Z^{1 / 2},
\end{aligned}
$$

where $\overline{t^{(3)}\left(\mathbf{u}_{1}, \mathbf{u}_{2}\right)}$ is the average OTF over the mid frequency and $\sim 5 \times 10^{-2}$. $\quad \bar{n}$ can be expressed as functions of the magnitude of the object $m$, the fractional bandwidth $\Delta \lambda / \lambda$, the efficiency of the detection system $\eta$, the integration time $\Delta \tau$, and the coherence length $\mathbf{r}_{c}$. The limiting magnitude at $\lambda=0.55 \mu \mathrm{m}$ is given as

$$
\begin{aligned}
m_{\lim }= & 13.3+2.5\left\{\log \left(\frac{\Delta \lambda / \lambda}{0.1}\right)+\log \left(\frac{\eta}{0.1}\right)+\log \left(\frac{\Delta \tau}{10 \mathrm{msec}}\right)\right. \\
& +\frac{4}{3} \log \left(\frac{r_{\mathrm{c}}}{14 \mathrm{~cm}}\right)+\frac{2}{3} \log \left(\frac{D}{1 \mathrm{~m}}\right)+\frac{1}{3} \log \left(\frac{Z}{10^{4}}\right) \\
& \left.-\frac{2}{3} \log \left[\frac{\mathrm{SNR}(\mathrm{map})}{10}\right]\right\} .
\end{aligned}
$$

With a resolution approximately a factor of 2 lower than the diffraction limit, a SNR of 10 is obtained from the attainable number of frames of $10^{4}$ for a point source of 13.3 magnitudes with a 1-m telescope and for a point source of 14.5 magnitudes with a 5-m telescope. For a good observing condition, $\Delta \tau$ may be somewhat longer, and the limiting magnitude may reach 15 mag. As can be seen from Fig. 10, the SNR at frequencies above $0.5 \mathrm{D} / \lambda$ decreases drastically according to the behavior of the OTF.

For the high-frequency region the nonredundant-masking method $10,24,25$ is more promising than the fully filled aperture method of the conventional speckle. From the interferometric view, for a certain Fourier component, other Fourier components behave as backgrounds. In the presence of overwhelming lower-frequency components, high-frequency components are suppressed strongly because of the low redundancy of long baselines. However, before we proceed to a quantitative comparison between the fully filled-aperture method and the nonredundant-masking method, there are still problems to be solved, such as the estimation of the independent bispectral volume for the nonredundant masking. ${ }^{26}$

\section{CONCLUSIONS}

In this paper the behavior of the SNR of the bispectral analysis of speckle interferometry is studied in two stages. At the high-light limit, the Monte Carlo simulations of an atmospheric phase screen based on the Kolmogorov theory and recent observations of the atmospheric disturbance are used to derive statistical properties of the classical bispectral MTF. The influence of photon noise is taken into account by modeling the photodetection process.

A general expression for the SNR of the bispectrum at arbitrary light levels is obtained in terms of the classical MTF's and the mean photon counts. In the mid-frequency range, a practical expression is obtained for the SNR as a function of the OTF's of the telescope optics, the number of speckles, and the mean photon counts.

Major conclusions are as follows:

(1) The overall behavior of the MTF is qualitatively consistent with the HIV of the image-forming process, and, especially in the mid-frequency range, the quantitative predictions of the HIV agree approximately with the simulated results. At the mid frequencies, the attenuation of the bispectral MTF and the SNR are approximated by the predicted values $n_{s}{ }^{-2}$ and $n_{s}{ }^{-1 / 2}$, respectively.

(2) At low light levels, only bispectral components near the axes have a high SNR. Closure phases near the axes are effectively local phase differences. For simple sources, the behavior of the phase in Fourier space is so regular that local phase differences are enough for a full image recovery. In recovering complicated sources, global closure phases contained in the mid-frequency range may be crucial for the reconstruction of images of complicated sources. However, the SNR at the mid frequency falls off so drastically at low light levels that the effective limiting magnitudes are much lower than those of simple sources.

(3) As estimated from the SNR in the mid-frequency range, the practical limiting magnitude of the bispectral analysis at a visual wavelength is between 13 and 15 magnitudes, depending on the size of the telescope and the observing conditions. This limit is achieved with a resolution that is half the diffraction limit of a given telescope. 


\section{APPENDIX A: PREDICTIONS OBTAINED BY THE TREATMENT BASED ON THE HEURISTIC INTERFEROMETRIC VIEW OF THE IMAGE- FORMING PROCESS}

A detailed treatment based on the HIV of the image-forming process was discussed in Ref. 10. In this appendix the major predictions are reviewed briefly.

A speckle pattern is regarded as an instantaneous interference pattern formed by a number of elementary coherent areas on the aperture plane, whose linear sizes are about $r_{c}$. The discussion must be restricted to mid spatial frequency, where a certain Fourier component on the image plane is given as a sum of random phasors originating from identical baselines on the aperture plane. A mid-frequency component satisfies the following two conditions. First, the corresponding baselines to a mid-frequency component are so much longer than $r_{c}$ that the rms phase $\left(\sigma_{\Phi}\right)$ of the baselines is significantly larger than $2 \pi$. In other words, the rms phase correlation function of a pair of elementary areas is significantly larger than $2 \pi$. The unit phasor $\exp (i \Phi)$ of a baseline then becomes a uniform random number on the unit circle on the complex plane. Effectively a $\Phi$ can be regarded as a uniform random number between $-\pi$ and $\pi$. Second, the redundancy of the baseline must be high so that the number of random phasors is large enough for an incoherent average to be performed. Although individual phasors have uniformly random phases, phases of neighboring baselines are correlated. To ensure that a good average is obtained over the random phasors, the number of phasors must be significantly larger than $2 \pi$. (Recall that the translation by $r_{c}$ on the aperture plane causes a rms phase change of $1 \mathrm{rad}$.) At the highest-frequency region of a circular aperture, this condition is not satisfied; thus we restrict our discussion to the mid-frequency range.

The dependences of the power-spectrum ATF, the bispectral ATF, and their SNR's on the number of speckles $\left[n_{s}=\right.$ $\left.\left(D / r_{c}\right)^{2}\right]$ are determined below. For simplicity, we neglect scintillation and assume unit phasors originating from individual baselines.

$\mathbf{u}_{1}, \tilde{I}\left(\mathbf{u}_{1}\right)$, and $N\left(\mathbf{u}_{1}\right)$ denote a mid-spatial frequency and the Fourier component and the number of phasors or redundancy of the baseline corresponding to $\mathbf{u}_{1}$, respectively. The Fourier component is given as

$$
\tilde{I}\left(\mathbf{u}_{1}\right)=\sum_{k=1}^{N\left(\mathbf{u}_{1}\right)} \exp \left(i \Phi_{k}\right)
$$

where $\Phi_{k}$ is the phase of the $k$ th phasor. The average powerspectrum MTF over an ensemble of speckle patterns is

$$
\begin{aligned}
\left\langle\left|\tilde{I}\left(\mathbf{u}_{1}\right)\right|^{2}\right\rangle & =\left\langle\sum_{k=1}^{N\left(\mathbf{u}_{1}\right)} \sum_{l=1}^{N\left(\mathbf{u}_{1}\right)} \exp \left[i\left(\Phi_{k}-\Phi_{l}\right)\right]\right\rangle \\
& =\sum_{k=1}^{N\left(\mathbf{u}_{1}\right)} \sum_{l=1}^{N\left(\mathbf{u}_{1}\right)}\left\langle\exp \left[i\left(\Phi_{k}-\Phi_{l}\right)\right]\right\rangle .
\end{aligned}
$$

Since $\Phi_{k}$ and $\Phi_{l}$ are not correlated unless $k=l$,

$$
\begin{array}{rlrl}
\left\langle\exp \left[i\left(\Phi_{k}-\Phi_{l}\right)\right]\right\rangle & =1, & & k=l, \text { with } N\left(\mathbf{u}_{1}\right) \text { terms } \\
& =0, & & k \neq l, \text { with } N\left(\mathbf{u}_{1}\right)\left[N\left(\mathbf{u}_{1}\right)-1\right] \\
& \text { terms; }
\end{array}
$$

then

$$
\left\langle\left|\tilde{I}\left(\mathbf{u}_{1}\right)\right|^{2}\right\rangle=N\left(\mathbf{u}_{1}\right)
$$

In the absence of atmospheric disturbance,

$$
\left|\tilde{I}\left(\mathbf{u}_{1}\right)\right|^{2}=N\left(\mathbf{u}_{1}\right)^{2}
$$

Thus the ATF or the power-spectrum ATF is $N\left(\mathbf{u}_{1}\right)^{-1}$. In addition to the ATF that is due to the atmosphere, there is an atmospheric noise factor that is $\sim N\left(\mathbf{u}_{1}\right)$. Thus the SNR of $\left\langle\left|\tilde{I}\left(\mathbf{u}_{1}\right)\right|^{2}\right\rangle$ is 1 . In the mid-frequency region, the redundancy $N(\mathbf{u})$ is proportional to and of the order of the number of speckles, $n_{s}=\left(D / r_{c}\right)^{2}$. The ATF is about $n_{s}{ }^{-1}$.

The bispectral ATF is obtained in the same manner. The ensemble average of the bispectral component at $\left(\mathbf{u}_{1}, \mathbf{u}_{2}\right)$ is

$$
\begin{aligned}
\left\langle\tilde{I}^{(3)}\left(\mathbf{u}_{1}, \mathbf{u}_{2}\right)\right\rangle & \\
= & \left\langle\sum_{k=1}^{N\left(\mathbf{u}_{1}\right)} \exp \left(i \Phi_{12, k}\right) \sum_{l=1}^{N\left(\mathbf{u}_{2}\right)} \exp \left(i \Phi_{23, l}\right) \cdot \sum_{m=1}^{N\left(-\mathbf{u}_{1}-\mathbf{u}_{2}\right)} \exp \left(i \Phi_{31, m}\right)\right\rangle \\
= & \sum_{k=1}^{N\left(\mathbf{u}_{1}\right)} \sum_{l=1}^{N\left(\mathbf{u}_{2}\right)} \sum_{m=1}^{N\left(-\mathbf{u}_{1}-\mathbf{u}_{2}\right)}\left\langle\exp \left[i\left(\Phi_{12, k}+\Phi_{23, l}+\Phi_{31, m}\right)\right]\right\rangle,
\end{aligned}
$$

where 12,23 , and 31 denote baselines corresponding to the frequencies $\mathbf{u}_{1}, \mathbf{u}_{2}$, and $-\mathbf{u}_{1}-\mathbf{u}_{2}$, respectively. The only terms with $k=l=m$ have finite contributions, and the other terms have zero mean. In the ideal case in which the closure-phase cancellation is perfect,

$$
\left\langle\tilde{I}^{(3)}\left(\mathbf{u}_{1}, \mathbf{u}_{2}\right)\right\rangle=\operatorname{Min}\left[N\left(\mathbf{u}_{1}\right), N\left(\mathbf{u}_{2}\right), N\left(-\mathbf{u}_{1}-\mathbf{u}_{2}\right)\right],
$$

where $\operatorname{Min}\left[N\left(\mathbf{u}_{1}\right), N\left(\mathbf{u}_{2}\right), N\left(-\mathbf{u}_{1}-\mathbf{u}_{2}\right)\right]$ is the minimum among the three redundancies and the number of closed triangles. In the absence of atmospheric disturbance,

$$
\tilde{I}^{(3)}\left(\mathbf{u}_{1}, \mathbf{u}_{2}\right)=N\left(\mathbf{u}_{1}\right) N\left(\mathbf{u}_{2}\right) N\left(-\mathbf{u}_{1}-\mathbf{u}_{2}\right) ;
$$

then the bispectral ATF at $\left(\mathbf{u}_{1}, \mathbf{u}_{2}\right)$ is

$$
\frac{\operatorname{Min}\left[N\left(\mathbf{u}_{1}\right), N\left(\mathbf{u}_{2}\right), N\left(-\mathbf{u}_{1}-\mathbf{u}_{2}\right)\right]}{N\left(\mathbf{u}_{1}\right) N\left(\mathbf{u}_{2}\right) N\left(-\mathbf{u}_{1}-\mathbf{u}_{2}\right)} .
$$

After $N\left(\mathbf{u}_{1}\right) N\left(\mathbf{u}_{2}\right) N\left(-\mathbf{u}_{1}-\mathbf{u}_{2}\right)$ terms are added, the average becomes $\operatorname{Min}\left[N\left(\mathbf{u}_{1}\right), N\left(\mathbf{u}_{2}\right), N\left(-\mathbf{u}_{1}-\mathbf{u}_{2}\right)\right]$. Therefore the SNR is

$$
\frac{\operatorname{Min}\left[N\left(\mathbf{u}_{1}\right), N\left(\mathbf{u}_{2}\right), N\left(-\mathbf{u}_{1}-\mathbf{u}_{2}\right)\right]}{\left[N\left(\mathbf{u}_{1}\right) N\left(\mathbf{u}_{2}\right) N\left(-\mathbf{u}_{1}-\mathbf{u}_{2}\right)\right]^{1 / 2}} .
$$

Estimates of the bispectral ATF and the SNR are given as $n_{s}{ }^{-2}$ and $n_{s}{ }^{-1 / 2}$, respectively.

In what follows, higher-order ATF's used to derive Eqs. (7.27)-(7.30) are calculated. 
$\left\langle\tilde{a}^{(4)}\left(\mathbf{u}_{1}, \mathbf{u}_{2}, \mathbf{u}_{3}\right)\right\rangle$

$N\left(\mathbf{u}_{1}\right) N\left(\mathbf{u}_{1}\right) N\left(\mathbf{u}_{3}\right) N\left(-\mathbf{u}_{1}-\mathbf{u}_{2}-\mathbf{u}_{3}\right)$

$=\frac{\sum_{k=1} \sum_{l=1} \sum_{m=1} \sum_{n=1}\left\langle\exp \left[\tilde{i}\left(\Phi_{12, k}+\Phi_{23, l}+\Phi_{34, m}+\Phi_{41, n}\right)\right]\right\rangle}{N\left(\mathbf{u}_{1}\right) N\left(\mathbf{u}_{2}\right) N\left(\mathbf{u}_{3}\right) N\left(-\mathbf{u}_{1}-\mathbf{u}_{2}-\mathbf{u}_{3}\right)}$

$=\frac{\operatorname{Min}\left[N\left(\mathbf{u}_{1}\right), N\left(\mathbf{u}_{2}\right), N\left(\mathbf{u}_{3}\right), N\left(-\mathbf{u}_{1}-\mathbf{u}_{2}-\mathbf{u}_{3}\right)\right]}{N\left(\mathbf{u}_{1}\right) N\left(\mathbf{u}_{2}\right) N\left(\mathbf{u}_{3}\right) N\left(-\mathbf{u}_{1}-\mathbf{u}_{2}-\mathbf{u}_{3}\right)}$

$\approx n_{s}^{-3}$, $\left\langle\left|\tilde{a}\left(\mathbf{u}_{1}\right)\right|^{2}\left|\tilde{a}\left(\mathbf{u}_{2}\right)\right|^{2}\right\rangle$

$$
=\frac{\sum_{k=1}^{N\left(\mathbf{u}_{1}\right)} \sum_{l=1}^{N\left(\mathbf{u}_{1}\right)} \sum_{m=1}^{N\left(\mathbf{u}_{2}\right)} \sum_{n=1}^{N\left(\mathbf{u}_{2}\right)}\left\langle\exp \left[i\left(\Phi_{12, k}-\Phi_{12, l}+\Phi_{34, m}-\Phi_{34, n}\right)\right]\right\rangle}{N\left(\mathbf{u}_{1}\right)^{2} N\left(\mathbf{u}_{2}\right)^{2}}
$$$$
=\frac{N\left(\mathbf{u}_{1}\right) N\left(\mathbf{u}_{2}\right)}{N\left(\mathbf{u}_{1}\right)^{2} N\left(\mathbf{u}_{2}\right)^{2}}
$$$$
\approx n_{s}^{-2} \text {, }
$$

since for only the terms with $k=l, m=n$,

$$
\left\langle\exp \left[i\left(\Phi_{12, k}-\Phi_{12, l}+\Phi_{34, m}-\Phi_{34, n}\right)\right]\right\rangle=1 ;
$$

$$
\begin{aligned}
\left\langle\left|\tilde{a}\left(\mathbf{u}_{1}\right)\right|^{2} \tilde{a}^{(3)}\left(\mathbf{u}_{2}, \mathbf{u}_{3}\right)\right\rangle & =\sum_{k=1}^{N\left(\mathbf{u}_{1}\right)} \sum_{l=1}^{N\left(\mathbf{u}_{1}\right)} \sum_{m=1}^{N\left(\mathbf{u}_{i}\right)} \sum_{n=1}^{N\left(\mathbf{u}_{3}\right)} \sum_{o=1}^{N\left(-\mathbf{u}_{2}-\mathbf{u}_{3}\right)}\left\langle\exp \left[i\left(\Phi_{12, k}-\Phi_{12, l}+\Phi_{34, m}+\Phi_{45, n}+\Phi_{53, o}\right)\right]\right\rangle / N\left(\mathbf{u}_{1}\right)^{2} N\left(\mathbf{u}_{2}\right) N\left(\mathbf{u}_{3}\right) N\left(-\mathbf{u}_{2}-\mathbf{u}_{3}\right) \\
& =\frac{N\left(\mathbf{u}_{1}\right) \times \operatorname{Min}\left[N\left(\mathbf{u}_{2}\right), N\left(\mathbf{u}_{3}\right), N\left(-\mathbf{u}_{2}-\mathbf{u}_{3}\right)\right]}{N\left(\mathbf{u}_{1}\right)^{2} N\left(\mathbf{u}_{2}\right) N\left(\mathbf{u}_{3}\right) N\left(-\mathbf{u}_{2}-\mathbf{u}_{3}\right)} \\
& \approx n_{s}{ }^{-3}
\end{aligned}
$$

since for only the terms with $k=l, m=n=0$,

$$
\left\langle\exp \left[i\left(\Phi_{23, k}-\Phi_{12, l}+\Phi_{34, m}+\Phi_{45, n}+\Phi_{53, o}\right)\right]\right\rangle=1,
$$

$$
\begin{aligned}
\left\langle\left|\tilde{a}^{(3)}\left(\mathbf{u}_{1}, \mathbf{u}_{2}\right)\right|^{2}\right\rangle= & \sum_{k=1}^{N\left(\mathbf{u}_{1}\right)} \sum_{l=1}^{N\left(\mathbf{u}_{2}\right)} \sum_{m=1}^{N\left(-\mathbf{u}_{1}-\mathbf{u}_{2}\right)} \sum_{n=1}^{N\left(\mathbf{u}_{1}\right)} \sum_{o=1}^{N\left(\mathbf{u}_{2}\right)} \sum_{p=1}^{\left(-\mathbf{u}_{1}-\mathbf{u}_{2}\right)} \\
& \left\langle\operatorname { e x p } \left[i \left(\Phi_{12, k}+\Phi_{23, l}+\Phi_{31, m}-\Phi_{12, n}-\Phi_{23, o}\right.\right.\right. \\
& \left.\left.\left.-\Phi_{31, p}\right)\right]\right\rangle / N\left(\mathbf{u}_{1}\right)^{2} N\left(\mathbf{u}_{2}\right)^{2} N\left(-\mathbf{u}_{1}-\mathbf{u}_{2}\right)^{2} \\
= & \frac{N\left(\mathbf{u}_{1}\right) N\left(\mathbf{u}_{2}\right) N\left(-\mathbf{u}_{1}-\mathbf{u}_{2}\right)+\operatorname{Min}\left[N\left(\mathbf{u}_{2}\right), N\left(\mathbf{u}_{3}\right), N\left(-\mathbf{u}_{2}-\mathbf{u}_{3}\right)\right]^{2}}{N\left(\mathbf{u}_{1}\right)^{2} N\left(\mathbf{u}_{3}\right)^{2} N\left(-\mathbf{u}_{1}-\mathbf{u}_{2}\right)^{2}} \\
\approx & n_{s}{ }^{-3} ;
\end{aligned}
$$

since the following closure-phase cancellations work only for the terms with $k=l=m=n$ :

$$
\begin{aligned}
\Phi_{12, k}+\Phi_{23, k}+\Phi_{34, k}+\Phi_{41, k}= & \left(\Phi_{12, k}+\Phi_{23, k}+\Phi_{31, k}\right) \\
& +\left(\Phi_{13, k}+\Phi_{34, k}+\Phi_{41, k}\right) \\
= & 0 .
\end{aligned}
$$

Thus we have since for only the terms with $k=n, l=o, m=p$ or $k=l=m, o$ $=p=q$,

$$
\left\langle\exp \left[i\left(\Phi_{12, k}+\Phi_{23, l}+\Phi_{31, m}-\Phi_{12, n}-\Phi_{23, o}-\Phi_{31, p}\right)\right]\right\rangle=1 .
$$

\section{APPENDIX B: CALCULATIONS OF THE VARIANCE OF THE BISPECTRUM}

In the estimation of the expected value of the variance, the starting point is the modulus squared of Eq. (7.21):

$$
\begin{array}{r}
E_{\alpha \beta \gamma \delta \epsilon \zeta}\left(\sum_{\alpha \neq \beta \neq \gamma} \sum_{\delta \neq \epsilon \neq \zeta} \exp \left\{i\left[\mathbf{u}_{1}\left(\mathbf{x}_{\alpha}-\mathbf{x}_{\gamma}-\mathbf{x}_{\delta}+\mathbf{x}_{\zeta}\right)+\mathbf{u}_{2}\left(\mathbf{x}_{\beta}-\mathbf{x}_{\gamma}-\mathbf{x}_{\epsilon}+\mathbf{x}_{\zeta}\right)\right]\right\}\right) \\
=\sum_{\alpha \neq \beta \neq \gamma} \sum_{\delta \neq \epsilon \neq \zeta} E_{\alpha \beta \gamma \delta \epsilon \zeta}\left(\exp \left\{i\left[\mathbf{u}_{1}\left(\mathbf{x}_{\alpha}-\mathbf{x}_{\gamma}-\mathbf{x}_{\delta}+\mathbf{x}_{\zeta}\right)+\mathbf{u}_{2}\left(\mathbf{x}_{\beta}-\mathbf{x}_{\gamma}-\mathbf{x}_{\epsilon}+\mathbf{x}_{\zeta}\right)\right]\right\}\right)
\end{array}
$$


where $E_{\alpha \beta \gamma \delta \epsilon \zeta}$ denotes an average over $\mathbf{x}_{\alpha}, \mathbf{x}_{\beta}, \mathbf{x}_{\gamma}, \mathbf{x}_{\delta}, \mathbf{x}_{\epsilon}$, and $\mathbf{x}_{j}$. The $\left[N_{j}\left(N_{j}-1\right)\left(N_{j}-2\right)\right]^{2}$ terms are classified as follows:

(1) For $\alpha=\delta, \beta=\epsilon, \gamma=\zeta$ and $N_{j}\left(N_{j}-1\right)\left(N_{j}-2\right)$ terms,

$$
E_{\alpha \beta \gamma \delta \epsilon \zeta}(1)=1 \text {. }
$$

(2) For $\alpha=\epsilon, \beta=\delta, \gamma=\zeta$ and $N_{j}\left(N_{j}-1\right)\left(N_{j}-2\right)$ terms, $E_{\alpha \beta \gamma \delta \epsilon}\left\{\exp \left[i\left(\mathbf{u}_{1}-\mathbf{u}_{2}\right)\left(\mathbf{x}_{\alpha}-\mathbf{x}_{\beta}\right)\right]\right\}=\left|\tilde{i}_{j}\left(\mathbf{u}_{1}-\mathbf{u}_{2}\right)\right|^{2}$.

(3) For $\alpha=\zeta, \beta=\epsilon, \gamma=\delta$ and $N_{j}\left(N_{j}-1\right)\left(N_{j}-2\right)$ terms, $E_{\alpha \beta \gamma \delta \epsilon}\left\{\exp \left[i\left(2 \mathbf{u}_{1}+\mathbf{u}_{2}\right)\left(\mathbf{x}_{\alpha}-\mathbf{x}_{\gamma}\right)\right]\right\}=\left|\tilde{i}_{j}\left(2 \mathbf{u}_{1}+\mathbf{u}_{2}\right)\right|^{2}$.

(4) For $\alpha=\delta, \beta=\zeta, \gamma=\epsilon$ and $N_{j}\left(N_{j}-1\right)\left(N_{j}-2\right)$ terms, $E_{\alpha \beta \gamma \delta \epsilon}\left\{\exp \left[i\left(\mathbf{u}_{1}+2 \mathbf{u}_{2}\right)\left(\mathbf{x}_{\beta}-\mathbf{x}_{\gamma}\right)\right]\right\}=\left|\tilde{i}_{j}\left(\mathbf{u}_{1}+2 \mathbf{u}_{2}\right)\right|^{2}$.

(5) For $\alpha=\epsilon, \beta=\zeta, \gamma=\delta$ and $N_{j}\left(N_{j}-1\right)\left(N_{j}-2\right)$ terms, $E_{\alpha \beta \gamma \delta \epsilon \zeta}\left(\exp \left\{i\left[\left(\mathbf{u}_{1}-\mathbf{u}_{2}\right) \mathbf{x}_{\alpha}+\left(\mathbf{u}_{1}+2 \mathbf{u}_{2}\right) \mathbf{x}_{\beta}+\left(-2 \mathbf{u}_{1}-\mathbf{u}_{2}\right) \mathbf{x}_{\gamma}\right]\right\}\right)$ $=\tilde{b}_{j}^{(3)}\left(\mathbf{u}_{1}-\mathbf{u}_{2}, \mathbf{u}_{1}+2 \mathbf{u}_{2}\right)$.

(6) For $\alpha=\zeta, \beta=\delta, \gamma=\epsilon$ and $N_{j}\left(N_{j}-1\right)\left(N_{j}-2\right)$ terms, $E_{\alpha \beta \gamma \delta \epsilon}\left(\exp \left\{i\left[\left(2 \mathbf{u}_{1}+\mathbf{u}_{2}\right) \mathbf{x}_{\alpha}+\left(-\mathbf{u}_{1}+\mathbf{u}_{2}\right) \mathbf{x}_{\beta}+\left(-\mathbf{u}_{1}-2 \mathbf{u}_{2}\right) \mathbf{x}_{\gamma}\right]\right\}\right)$

$$
=\tilde{b}_{j}^{(3)}\left(2 \mathbf{u}_{1}+\mathbf{u}_{2},-\mathbf{u}_{1}+\mathbf{u}_{2}\right) \text {. }
$$

(7) For $\alpha \neq \delta, \beta=\epsilon, \gamma=\zeta$ and $N_{j}\left(N_{j}-1\right)\left(N_{j}-2\right)\left(N_{j}-3\right)$ terms,

$$
E_{\alpha \beta \gamma \delta \epsilon\}}\left\{\exp \left[i \mathbf{u}_{1}\left(\mathbf{x}_{\alpha}-\mathbf{x}_{\delta}\right)\right]\right\}=\left|\tilde{i}_{j}\left(\mathbf{u}_{1}\right)\right|^{2}
$$

(8) For $\alpha=\delta, \beta \neq \epsilon, \gamma=\zeta$ and $N_{j}\left(N_{j}-1\right)\left(N_{j}-2\right)\left(N_{j}-3\right)$ terms,

$$
E_{\alpha \beta \gamma \delta \epsilon\}}\left\{\exp \left[i \mathbf{u}_{2}\left(\mathbf{x}_{\beta}-\mathbf{x}_{\epsilon}\right)\right]\right\}=\left|\tilde{i}_{j}\left(\mathbf{u}_{2}\right)\right|^{2}
$$

(9) For $\alpha=\delta, \beta=\epsilon, \gamma \approx \zeta$ and $N_{j}\left(N_{j}-1\right)\left(N_{j}-2\right)\left(N_{j}-3\right)$ terms,

$$
E_{\alpha \beta \gamma \delta \epsilon}\left\{\exp \left[i\left(-\mathbf{u}_{1}-\mathbf{u}_{2}\right)\left(\mathbf{x}_{\gamma}-\mathbf{x}_{\zeta}\right)\right]\right\}=\left|\tilde{i}_{j}\left(-\mathbf{u}_{1}-\mathbf{u}_{2}\right)\right|^{2} .
$$

(10) For $\alpha \neq \epsilon, \beta=\delta, \gamma=\zeta$ and $N_{j}\left(N_{j}-1\right)\left(N_{j}-2\right)\left(N_{j}-\right.$ 3) terms,

$$
E_{\alpha \beta \gamma \delta \xi \zeta}\left(\exp \left\{i\left[\mathbf{u}_{1} \mathbf{x}_{\alpha}+\left(-\mathbf{u}_{1}+\mathbf{u}_{2}\right) \mathbf{x}_{\beta}-\mathbf{u}_{2} \mathbf{x}_{\epsilon}\right]\right\}\right)=\tilde{b}_{j}^{(3)}\left(\mathbf{u}_{1},-\mathbf{u}_{2}\right) .
$$

(11) For $\alpha=\epsilon, \beta \neq \delta, \gamma=\zeta$ and $N_{j}\left(N_{j}-1\right)\left(N_{j}-2\right)\left(N_{j}-\right.$ 3) terms,

$$
\begin{aligned}
E_{\alpha \beta \gamma \delta \epsilon \zeta}\left(\exp \left\{i\left[\left(\mathbf{u}_{1}-\mathbf{u}_{2}\right) \mathbf{x}_{\alpha}+\mathbf{u}_{2} \mathbf{x}_{\beta}-\mathbf{u}_{1} \mathbf{x}_{\delta}\right]\right\}\right) & =\tilde{b}_{j}^{(3)}\left(-\mathbf{u}_{1}, \mathbf{u}_{2}\right) \\
& =\tilde{b}_{j}^{(3)}\left(\mathbf{u}_{1},-\mathbf{u}_{2}\right)^{*}
\end{aligned}
$$

(12) For $\alpha=\delta, \beta \neq \zeta, \gamma=\epsilon$ and $N_{j}\left(N_{j}-1\right)\left(N_{j}-2\right)\left(N_{j}-\right.$ 3) terms,

$$
\begin{aligned}
E_{\alpha \beta \gamma \delta \zeta}\left(\operatorname { e x p } \left\{i \left[\mathbf{u}_{2} \mathbf{x}_{\beta}-\left(\mathbf{u}_{1}+2 \mathbf{u}_{2}\right) \mathbf{x}_{\gamma}+\left(\mathbf{u}_{1}\right.\right.\right.\right. & \left.\left.\left.\left.+\mathbf{u}_{2}\right) \mathbf{x}_{\zeta}\right\}\right\}\right) \\
& =\tilde{b}_{j}^{(3)}\left(\mathbf{u}_{1}+\mathbf{u}_{2}, \mathbf{u}_{2}\right) .
\end{aligned}
$$

(13) For $\alpha=\delta, \beta=\zeta, \gamma \neq \epsilon$ and $N_{j}\left(N_{j}-1\right)\left(N_{j}-2\right)\left(N_{j}-\right.$ 3) terms,

$$
\begin{aligned}
E_{\alpha \beta \gamma \delta \epsilon \zeta}\left(\operatorname { e x p } \left\{i \left[\left(\mathbf{u}_{1}+2 \mathbf{u}_{2}\right) \mathbf{x}_{\beta}-\left(\mathbf{u}_{1}+\mathbf{u}_{2}\right) \mathbf{x}_{\gamma}\right.\right.\right. & \left.\left.\left.+\mathbf{u}_{2} \mathbf{x}_{\epsilon}\right]\right\}\right) \\
& =\tilde{b}_{j}^{(3)}\left(-\mathbf{u}_{1}-\mathbf{u}_{2}, \mathbf{u}_{2}\right) \\
& =\tilde{b}_{j}^{(3)}\left(\mathbf{u}_{1}+\mathbf{u}_{2}, \mathbf{u}_{2}\right)^{*} .
\end{aligned}
$$

(14) For $\alpha \neq \zeta, \beta=\epsilon, \gamma=\delta$ and $N_{j}\left(N_{j}-1\right)\left(N_{j}-2\right)\left(N_{j}-\right.$ 3) terms,

$$
\begin{aligned}
E_{\alpha \beta \gamma \delta \epsilon \zeta}\left(\operatorname { e x p } \left\{i \left[\mathbf{u}_{1} \mathbf{x}_{\alpha}-\left(2 \mathbf{u}_{1}+\mathbf{u}_{2}\right) \mathbf{x}_{\gamma}+\left(\mathbf{u}_{1}+\right.\right.\right.\right. & \left.\left.\left.\left.\mathbf{u}_{2}\right) \mathbf{x}_{\zeta}\right]\right\}\right) \\
& =\tilde{b}_{j}^{(3)}\left(\mathbf{u}_{1}+\mathbf{u}_{2}, \mathbf{u}_{1}\right) .
\end{aligned}
$$

(15) For $\alpha=\zeta, \beta=\epsilon, \gamma \neq \delta$ and $N_{j}\left(N_{j}-1\right)\left(N_{j}-2\right)\left(N_{j}-\right.$ 3) terms,

$E_{\alpha \beta \gamma \delta \epsilon \zeta}\left(\exp \left\{i\left[\left(2 \mathbf{u}_{1}+\mathbf{u}_{2}\right) \mathbf{x}_{\alpha}-\left(\mathbf{u}_{1}+\mathbf{u}_{2}\right) \mathbf{x}_{\gamma}-\mathbf{u}_{1} \mathbf{x}_{\delta}\right]\right\}\right)$

$$
\begin{aligned}
& =\tilde{b}_{j}^{(3)}\left(-\mathbf{u}_{1}-\mathbf{u}_{2},-\mathbf{u}_{1}\right) \\
& =\tilde{b}_{j}^{(3)}\left(\mathbf{u}_{1}+\mathbf{u}_{2}, \mathbf{u}_{1}\right)^{*}
\end{aligned}
$$

(16) For $\alpha \neq \delta, \beta=\zeta, \gamma=\epsilon$ and $N_{j}\left(N_{j}-1\right)\left(N_{j}-2\right)\left(N_{j}-\right.$ 3) terms,

$E_{\alpha \beta \gamma \delta \epsilon \zeta}\left(\exp \left\{i\left[\mathbf{u}_{1}\left(\mathbf{x}_{\alpha}-\mathbf{x}_{\delta}\right)+\left(\mathbf{u}_{1}+2 \mathbf{u}_{2}\right)\left(\mathbf{x}_{\beta}-\mathbf{x}_{\gamma}\right)\right]\right\}\right)$

$$
=\left|\tilde{i}_{j}\left(\mathbf{u}_{1}\right)\right|^{2}\left|\tilde{i}_{j}\left(\mathbf{u}_{1}+2 \mathbf{u}_{2}\right)\right|^{2} \text {. }
$$

(17) For $\alpha=\zeta, \beta \neq \epsilon, \gamma=\delta$ and $N_{j}\left(N_{j}-1\right)\left(N_{j}-2\right)\left(N_{j}-\right.$ 3) terms,

$E_{\alpha \beta \gamma \delta \epsilon \zeta}\left(\exp \left\{i\left[\mathbf{u}_{2}\left(\mathbf{x}_{\beta}-\mathbf{x}_{\epsilon}\right)+\left(2 \mathbf{u}_{1}+\mathbf{u}_{2}\right)\left(\mathbf{x}_{\alpha}-\mathbf{x}_{\gamma}\right)\right]\right\}\right)$

$$
=\left|\tilde{i}_{j}\left(\mathbf{u}_{2}\right)\right|^{2}\left|\tilde{i}_{j}\left(2 \mathbf{u}_{1}+\mathbf{u}_{2}\right)\right|^{2} \text {. }
$$

(18) For $\alpha=\epsilon, \beta=\delta, \gamma \neq \zeta$ and $N_{j}\left(N_{j}-1\right)\left(N_{j}-2\right)\left(N_{j}-\right.$ 3) terms,

$$
\begin{aligned}
E_{\alpha \beta \gamma \delta \epsilon \zeta}\left(\operatorname { e x p } \left\{i \left[\left(\mathbf{u}_{1}-\mathbf{u}_{2}\right)\left(\mathbf{x}_{\alpha}-\mathbf{x}_{\beta}\right)\right.\right.\right. & \left.\left.\left.-\left(\mathbf{u}_{1}+\mathbf{u}_{2}\right)\left(\mathbf{x}_{\gamma}-\mathbf{x}_{\zeta}\right)\right]\right\}\right) \\
& =\left|\tilde{i}_{j}\left(\mathbf{u}_{1}-\mathbf{u}_{2}\right)\right|^{2}\left|\tilde{i}_{j}\left(\mathbf{u}_{1}+\mathbf{u}_{2}\right)\right|^{2} .
\end{aligned}
$$

(19) For $\alpha=\epsilon, \beta=\zeta, \gamma \neq \delta$ and $N_{j}\left(N_{j}-1\right)\left(N_{j}-2\right)\left(N_{j}-\right.$ 3) terms,

$E_{\alpha \beta \gamma \delta \epsilon \zeta}\left(\exp \left\{i\left[\left(\mathbf{u}_{1}-\mathbf{u}_{2}\right) \mathbf{x}_{\alpha}+\left(\mathbf{u}_{1}+2 \mathbf{u}_{2}\right) \mathbf{x}_{\beta}-\left(\mathbf{u}_{1}+\mathbf{u}_{2}\right) \mathbf{x}_{\gamma}-\mathbf{u}_{1} \mathbf{x}_{\delta}\right]\right\}\right)$

$$
\begin{aligned}
& =\tilde{i}_{j}\left(\mathbf{u}_{1}-\mathbf{u}_{2}\right) \tilde{i}_{j}\left(\mathbf{u}_{1}+2 \mathbf{u}_{2}\right) \tilde{i}_{j}\left(-\mathbf{u}_{1}-\mathbf{u}_{2}\right) \tilde{i_{j}}\left(-\mathbf{u}_{1}\right) \\
& =\tilde{f}_{j}^{(4)}\left(\mathbf{u}_{1}-\mathbf{u}_{2}, \mathbf{u}_{1}+2 \mathbf{u}_{2},-\mathbf{u}_{1}-\mathbf{u}_{2}\right) .
\end{aligned}
$$

(20) For $\alpha \neq \zeta, \beta=\delta, \gamma=\epsilon$ and $N_{j}\left(N_{j}-1\right)\left(N_{j}-2\right)\left(N_{j}-\right.$ 3) terms,

$E_{\alpha \beta \gamma \delta \epsilon \zeta}\left(\exp \left\{i\left[\mathbf{u}_{1} \mathbf{x}_{\alpha}+\left(-\mathbf{u}_{1}+\mathbf{u}_{2}\right) \mathbf{x}_{\beta}-\left(\mathbf{u}_{1}+2 \mathbf{u}_{2}\right) \mathbf{x}_{\gamma}+\left(\mathbf{u}_{1}+\mathbf{u}_{2}\right) \mathbf{x}_{\delta}\right]\right\}\right)$

$$
\begin{aligned}
& =\tilde{i}_{j}\left(\mathbf{u}_{1}\right) \tilde{i}_{j}\left(-\mathbf{u}_{1}+\mathbf{u}_{2}\right) \tilde{i}_{j}\left(-\mathbf{u}_{1}-2 \mathbf{u}_{2}\right) \tilde{i}_{j}\left(\mathbf{u}_{1}+\mathbf{u}_{2}\right) \\
& =\tilde{f}_{j}^{(4)}\left(\mathbf{u}_{1}-\mathbf{u}_{2}, \mathbf{u}_{1}+2 \mathbf{u}_{2},-\mathbf{u}_{1}-\mathbf{u}_{2}\right)^{*} .
\end{aligned}
$$

(21) For $\alpha \neq \epsilon, \beta=\zeta, \gamma=\delta$ and $N_{j}\left(N_{j}-1\right)\left(N_{j}-2\right)\left(N_{j}-\right.$ 3) terms,

$$
\begin{array}{r}
E_{\alpha \beta \gamma \delta \epsilon}\left(\exp \left\{i\left[\mathbf{u}_{1} \mathbf{x}_{\alpha}+\left(\mathbf{u}_{1}+2 \mathbf{u}_{2}\right) \mathbf{x}_{\beta}-\left(2 \mathbf{u}_{1}+\mathbf{u}_{2}\right) \mathbf{x}_{\gamma}-\mathbf{u}_{2} \mathbf{x}_{\epsilon}\right]\right\}\right) \\
=\tilde{f}_{j}^{(4)}\left(\mathbf{u}_{1}, \mathbf{u}_{1}+2 \mathbf{u}_{2},-2 \mathbf{u}_{1}-\mathbf{u}_{2}\right) .
\end{array}
$$


(22) For $\alpha=\zeta, \beta \neq \delta, \gamma=\epsilon$ and $N_{j}\left(N_{j}-1\right)\left(N_{j}-2\right)\left(N_{j}-\right.$ 3) terms,

$$
\begin{aligned}
E_{\alpha \beta \gamma \delta \epsilon \zeta}\left(\operatorname { e x p } \left\{i \left[\left(2 \mathbf{u}_{1}+\mathbf{u}_{2}\right) \mathbf{x}_{\alpha}\right.\right.\right. & \left.\left.\left.+\mathbf{u}_{2} \mathbf{x}_{\beta}-\left(\mathbf{u}_{1}+2 \mathbf{u}_{2}\right) \mathbf{x}_{\gamma}-\mathbf{u}_{1} \mathbf{x}_{\delta}\right]\right\}\right) \\
& =\tilde{f}_{j}^{(4)}\left(\mathbf{u}_{1}, \mathbf{u}_{1}+2 \mathbf{u}_{2},-2 \mathbf{u}_{1}-\mathbf{u}_{2}\right)^{*} .
\end{aligned}
$$

(23) For $\alpha=\zeta, \beta=\delta, \gamma \neq \epsilon$ and $N_{j}\left(N_{j}-1\right)\left(N_{j}-2\right)\left(N_{j}-\right.$ 3) terms,

$$
\begin{array}{r}
E_{\alpha \beta \gamma \delta \hat{\epsilon}}\left(\exp \left\{i\left[\left(2 \mathbf{u}_{1}+\mathbf{u}_{2}\right) \mathbf{x}_{\alpha}+\left(-\mathbf{u}_{1}+\mathbf{u}_{2}\right) \mathbf{x}_{\beta}-\left(\mathbf{u}_{1}+\mathbf{u}_{2}\right) \mathbf{x}_{\gamma}-\mathbf{u}_{2} \mathbf{x}_{\epsilon}\right]\right\}\right) \\
=\tilde{f}_{j}^{(4)}\left(2 \mathbf{u}_{1}+\mathbf{u}_{2},-\mathbf{u}_{1}+\mathbf{u}_{2},-\mathbf{u}_{1}-\mathbf{u}_{2}\right) .
\end{array}
$$

(24) For $\alpha=\epsilon, \beta \neq \zeta, \gamma=\delta$ and $N_{j}\left(N_{j}-1\right)\left(N_{j}-2\right)\left(N_{j}-\right.$ 3) terms,

$E_{\alpha \beta \gamma \delta \epsilon \zeta}\left(\exp \left\{i\left[\left(\mathbf{u}_{1}-\mathbf{u}_{2}\right) \mathbf{x}_{\alpha}+\mathbf{u}_{2} \mathbf{x}_{\beta}+\left(-2 \mathbf{u}_{1}+\mathbf{u}_{2}\right) \mathbf{x}_{\gamma}+\left(\mathbf{u}_{1}+\mathbf{u}_{2}\right) \mathbf{x}_{\epsilon}\right]\right\}\right)$

$$
=\tilde{f}_{j}^{(4)}\left(2 \mathbf{u}_{1}+\mathbf{u}_{2},-\mathbf{u}_{1}+\mathbf{u}_{2},-\mathbf{u}_{1}-\mathbf{u}_{2}\right)^{*} \text {. }
$$

(25) For $\alpha \neq \delta, \beta \neq \epsilon, \gamma=\zeta$ and $N_{j}\left(N_{j}-1\right)\left(N_{j}-2\right)\left(N_{j}-\right.$ 3) $\left(N_{j}-4\right)$ terms,

$E_{\alpha \beta \gamma \delta \epsilon \zeta}\left(\exp \left\{i\left[\mathbf{u}_{1}\left(\mathbf{x}_{\alpha}-\mathbf{x}_{\delta}\right)+\mathbf{u}_{2}\left(\mathbf{x}_{\beta}-\mathbf{x}_{\epsilon}\right)\right]\right\}\right)=\left|\tilde{i}_{j}\left(\mathbf{u}_{1}\right)\right| 2\left|\tilde{i}_{j}\left(\mathbf{u}_{2}\right)\right|^{2}$.

(26) For $\alpha \neq \delta, \beta=\epsilon, \gamma \neq \zeta$ and $N_{j}\left(N_{j}-1\right)\left(N_{j}-2\right)\left(N_{j}-\right.$ 3) $\left(N_{j}-4\right)$ terms,

$$
\begin{aligned}
E_{\alpha \beta \gamma \delta \epsilon \zeta}\left(\operatorname { e x p } \left\{i \left[\mathbf{u}_{1}\left(\mathbf{x}_{\alpha}-\mathbf{x}_{\delta}\right)-\left(\mathbf{u}_{1}+\mathbf{u}_{2}\right)\right.\right.\right. & \left.\left.\left.\left(\mathbf{x}_{\gamma}-\mathbf{x}_{\zeta}\right)\right]\right\}\right) \\
& =\left|\tilde{i}_{j}\left(\mathbf{u}_{1}\right)\right|^{2}\left|\tilde{i}_{j}\left(-\mathbf{u}_{1}-\mathbf{u}_{2}\right)\right|^{2} .
\end{aligned}
$$

(27) For $\alpha=\delta, \beta \neq \epsilon, \gamma \neq \zeta$ and $N_{j}\left(N_{j}-1\right)\left(N_{j}-2\right)\left(N_{j}-\right.$ 3) $\left(N_{j}-4\right)$ terms,

$E_{\alpha \beta \gamma \delta \epsilon \zeta}\left(\exp \left\{i\left[\mathbf{u}_{2}\left(\mathbf{x}_{\beta}-\mathbf{x}_{\epsilon}\right)-\left(\mathbf{u}_{1}+\mathbf{u}_{2}\right)\left(\mathbf{x}_{\gamma}-\mathbf{x}_{\xi}\right)\right]\right\}\right)$

$$
=\left|\tilde{i}_{j}\left(\mathbf{u}_{2}\right)\right|^{2}\left|\tilde{i}_{j}\left(-\mathbf{u}_{1}-\mathbf{u}_{2}\right)\right|^{2} .
$$

(28) For $\alpha \neq \beta \neq \delta \neq \zeta, \gamma=\epsilon$ and $N_{j}\left(N_{j}-1\right)\left(N_{j}-2\right)\left(N_{j}\right.$ $-3)\left(N_{j}-4\right)$ terms,

$E_{\alpha \beta \gamma \delta \epsilon \zeta}\left(\exp \left\{i\left[\mathbf{u}_{1}\left(\mathbf{x}_{\alpha}-\mathbf{x}_{\delta}\right)+\mathbf{u}_{2}\left(\mathbf{x}_{\beta}-\mathbf{x}_{\gamma}\right)+\left(\mathbf{u}_{1}+\mathbf{u}_{2}\right)\left(\mathbf{x}_{\zeta}-\mathbf{x}_{\gamma}\right)\right]\right\}\right)$

$$
=\left|\tilde{i}_{j}\left(\mathbf{u}_{1}\right)\right|^{2} \tilde{b}_{j}^{(3)}\left(\mathbf{u}_{2}, \mathbf{u}_{1}+\mathbf{u}_{2}\right) \text {. }
$$

(29) For $\alpha \neq \gamma \neq \delta \neq \epsilon, \beta=\zeta$ and $N_{j}\left(N_{j}-1\right)\left(N_{j}-2\right)\left(N_{j}\right.$ $-3)\left(N_{j}-4\right)$ terms,

$E_{\alpha \beta \gamma \delta \epsilon \xi}\left(\exp \left\{i\left[\mathbf{u}_{1}\left(\mathbf{x}_{\alpha}-\mathbf{x}_{\delta}\right)-\mathbf{u}_{2}\left(\mathbf{x}_{\epsilon}-\mathbf{x}_{\beta}\right)-\left(\mathbf{u}_{1}+\mathbf{u}_{2}\right)\left(\mathbf{x}_{\gamma}-\mathbf{x}_{\beta}\right)\right]\right\}\right)$

$$
=\left|\tilde{i}_{j}\left(\mathbf{u}_{1}\right)\right|^{2} \tilde{b}_{j}^{(3)}\left(\mathbf{u}_{2}, \mathbf{u}_{1}+\mathbf{u}_{2}\right)^{*}
$$

(30) For $\alpha \neq \beta \neq \epsilon \neq \zeta, \gamma=\delta$ and $N_{j}\left(N_{j}-1\right)\left(N_{j}-2\right)\left(N_{j}\right.$ $-3)\left(N_{j}-4\right)$ terms,

$E_{\alpha \beta \gamma \delta \epsilon \zeta}\left(\exp \left\{i\left[\mathbf{u}_{2}\left(\mathbf{x}_{\beta}-\mathbf{x}_{\epsilon}\right)+\mathbf{u}_{1}\left(\mathbf{x}_{\alpha}-\mathbf{x}_{\gamma}\right)+\left(\mathbf{u}_{1}+\mathbf{u}_{2}\right)\left(\mathbf{x}_{\zeta}-\mathbf{x}_{\gamma}\right)\right]\right\}\right)$

$$
=\left|\tilde{i}_{j}\left(\mathbf{u}_{2}\right)\right|^{2} \tilde{b}_{j}^{(3)}\left(\mathbf{u}_{1}, \mathbf{u}_{1}+\mathbf{u}_{2}\right) \text {. }
$$

(31) For $\beta \neq \gamma \neq \neq \delta \neq \epsilon, \alpha=\zeta$ and $N_{j}\left(N_{j}-1\right)\left(N_{j}-2\right)\left(N_{j}\right.$ $-3)\left(N_{j}-4\right)$ terms,

$E_{\alpha \beta \gamma \delta \epsilon \zeta}\left(\exp \left\{i\left[\mathbf{u}_{2}\left(\mathbf{x}_{\beta}-\mathbf{x}_{\epsilon}\right)-\mathbf{u}_{1}\left(\mathbf{x}_{\delta}-\mathbf{x}_{\alpha}\right)-\left(\mathbf{u}_{1}+\mathbf{u}_{2}\right)\left(\mathbf{x}_{\gamma}-\mathbf{x}_{\alpha}\right)\right]\right\}\right)$

$$
=\left|\tilde{i}_{j}\left(\mathbf{u}_{2}\right)\right|^{2} \tilde{b}_{j}^{(3)}\left(\mathbf{u}_{1}, \mathbf{u}_{1}+\mathbf{u}_{2}\right)^{*} \text {. }
$$

(32) For $\alpha \neq \gamma \neq \epsilon \neq \zeta, \beta=\delta$ and $N_{j}\left(N_{j}-1\right)\left(N_{j}-2\right)\left(N_{j}\right.$ $-3)\left(N_{j}-4\right)$ terms,

$E_{\alpha \beta \gamma \delta \xi \xi}\left(\exp \left\{i\left[-\left(\mathbf{u}_{1}+\mathbf{u}_{2}\right)\left(\mathbf{x}_{\gamma}-\mathbf{x}_{\xi}\right)+\mathbf{u}_{1}\left(\mathbf{x}_{\alpha}-\mathbf{x}_{\beta}\right)-\mathbf{u}_{2}\left(\mathbf{x}_{\epsilon}-\mathbf{x}_{\beta}\right)\right]\right\}\right)$

$$
=\left|\tilde{i}_{j}\left(-\mathbf{u}_{1}-\mathbf{u}_{2}\right)\right|^{2} \tilde{b}_{j}^{(3)}\left(\mathbf{u}_{1},-\mathbf{u}_{2}\right) \text {. }
$$

(33) For $\beta \neq \gamma \neq \delta \neq \zeta, \alpha=\epsilon$ and $N_{j}\left(N_{j}-1\right)\left(N_{j}-2\right)\left(N_{j}\right.$ $-3)\left(N_{j}-4\right)$ terms,

$$
E_{\alpha \beta \gamma \delta \epsilon \zeta}\left(\exp \left\{i\left[-\left(\mathbf{u}_{1}+\mathbf{u}_{2}\right)\left(\mathbf{x}_{\gamma}-\mathbf{x}_{\zeta}\right)-\mathbf{u}_{1}\left(\mathbf{x}_{\delta}-\mathbf{x}_{\alpha}\right)+\mathbf{u}_{2}\left(\mathbf{x}_{\beta}-\mathbf{x}_{\alpha}\right)\right]\right\}\right)
$$$$
=\left|\tilde{i}_{j}\left(-\mathbf{u}_{1}-\mathbf{u}_{2}\right)\right|^{2} \tilde{b}_{j}^{(3)}\left(\mathbf{u}_{1},-\mathbf{u}_{2}\right)^{*} \text {. }
$$

(34) For $\alpha \neq \beta \neq \gamma \neq \delta \neq \epsilon \neq \zeta$ and $N_{j}\left(N_{j}-1\right)\left(N_{j}-\right.$ 2) $\left(N_{j}-3\right)\left(N_{j}-4\right)\left(N_{j}-5\right)$ terms,

$$
\begin{aligned}
E_{\alpha \beta \gamma \delta \epsilon \zeta}\left(\exp \left\{i\left[\mathbf{u}_{1}\left(\mathbf{x}_{\alpha}-\mathbf{x}_{\delta}\right)+\mathbf{u}_{2}\left(\mathbf{x}_{\beta}-\mathbf{x}_{\epsilon}\right)-\left(\mathbf{u}_{1}+\mathbf{u}_{2}\right)\left(\mathbf{x}_{\gamma}-\mathbf{x}_{\xi}\right)\right]\right\}\right) \\
=\left|\tilde{i}_{j}\left(\mathbf{u}_{1}\right)\right|{ }^{2}\left|\tilde{i}_{j}\left(\mathbf{u}_{2}\right)\right|^{2}\left|\tilde{i}_{j}\left(-\mathbf{u}_{1}-\mathbf{u}_{2}\right)\right|^{2} \\
=\left|\tilde{b}_{j}^{(3)}\left(\mathbf{u}_{1}, \mathbf{u}_{2}\right)\right|^{2}
\end{aligned}
$$

Averaging over the statistics of $N_{j}$ and $\lambda_{j}(\mathbf{x})$ and assuming that Eq. (7.16) holds, we obtain Eq. (7.22).

\section{APPENDIX C: VARIANCE FOR NON-PHOTON- COUNTING DETECTION}

For an observation with a non-photon-counting detector, it is impossible to remove bias terms frame by frame. An unbiased estimator of the classical bispectrum is again given by Eq. (7.20). However, fluctuations in the bias terms cause additional terms in the variance of the unbiased estimator of the classical bispectrum, Eq. (7.21). Those terms are evaluated as

$$
\begin{aligned}
& \bar{N}+\bar{N}^{2}+2\left(\bar{N}^{3}+2 \bar{N}^{2}\right)\left[\left\langle\left|\left(\mathbf{u}_{2}\right)\right|^{2}\right\rangle+\left\langle\left|\tilde{i}\left(\mathbf{u}_{2}\right)\right|^{2}\right\rangle+\left\langle\left|\tilde{i}\left(-\mathbf{u}_{1}-\mathbf{u}_{2}\right)\right|^{2}\right\rangle\right] \\
& \quad+\left(\bar{N}^{4}+3 \bar{N}^{3}\right)\left[\left\langle\tilde{b}^{(3)}\left(\mathbf{u}_{1}, \mathbf{u}_{2}\right)\right\rangle+\text { c.c. }\right]+\left(\bar{N}^{4}+4 \bar{N}^{3}\right. \\
& \left.\quad+2 \bar{N}^{2}\right)\left[\left\langle\left|\tilde{i}\left(\mathbf{u}_{1}\right)\right|^{4}\right\rangle+\left\langle\left|\tilde{i}\left(\mathbf{u}_{2}\right)\right|^{4}\right\rangle+\left\langle\left|\tilde{i}\left(-\mathbf{u}_{1}-\mathbf{u}_{2}\right)\right|^{4}\right\rangle\right. \\
& \quad+2\left\langle\left|\tilde{i}\left(\mathbf{u}_{1}\right)\right|^{2}\left|\tilde{i}\left(\mathbf{u}_{2}\right)\right|^{2}\right\rangle+\left\langle\left|\tilde{i}\left(\mathbf{u}_{2}\right)\right|^{2}\left|\tilde{i}\left(-\mathbf{u}_{1}-\mathbf{u}_{2}\right)\right|^{2}\right\rangle \\
& \left.\quad+\left\langle\left|\tilde{i}\left(-\mathbf{u}_{1}-\mathbf{u}_{2}\right)\right|^{2}\left|\tilde{i}\left(\mathbf{u}_{1}\right)\right|^{2}\right\rangle\right]+\left(\bar{N}^{5}+6 \bar{N}^{4}+6 \bar{N}^{3}\right) \\
& \left.\quad \times\left\langle\left[\tilde{b}^{(3)}\left(\mathbf{u}_{1}, \mathbf{u}_{2}\right)\right\rangle+\text { c.c. }\right]\left[\left|\tilde{i}\left(\mathbf{u}_{1}\right)\right|^{2}+\left|\tilde{i}\left(\mathbf{u}_{2}\right)\right|^{2}+\left|\tilde{i}\left(-\mathbf{u}_{1}-\mathbf{u}_{2}\right)\right|^{2}\right]\right\rangle .
\end{aligned}
$$

\section{ACKNOWLEDGMENTS}

It is a pleasure to express my special thanks to A. C. S. Readhead for initial suggestions on simulations, which were essential to this work. I would also thank P. W. Gohram, S. R. Kulkarni, G. Neugebauer, and A. C. S. Readhead for valuable discussions and useful comments on the manuscripts of the paper. I thank the editor, J. C. Dainty, and an anonymous referee for informing me of recent literature that was not mentioned in the original manuscript. Finally, I acknowledge the financial support of National Science Foundation grant AST 86-13059 and the Schlumberger Fellowship.

Note added in proof: While this paper was being processed, the author was informed by the editor that a recent work by Ayers et al. ${ }^{27}$ had some overlap with the present paper. Ayers et al. obtained, by means of a different logical path, an expression for the SNR of the bispectrum in the mid-frequency range that is identical to Eq. (7.34) of this paper. 


\section{REFERENCES}

1. A Labeyrie, "Attainment of diffraction-limited resolution in large telescopes by Fourier analysing speckle patterns in star images," Astron. Astrophys. 6, 85-87 (1970).

2. K. T. Knox and B. J. Thompson, "Recovery of images from atmospherically degraded short-exposure photographs," Astrophys. J. 193, L45-L48 (1974).

3. A. C. S. Readhead and P. N. Wilkinson, "The mapping of compact radio sources from VLBI data," Astrophys. J. 223, 25-36 (1978).

4. A. W. Lohmann, G. Weigelt, and B. Wirnitzer, "Speckle masking in astronomy: triple correlation theory and applications," Appl. Opt. 22, 4028-4037 (1983).

5. K.-H. Hofmann and G.Weigelt, "Speckle masking observation of the central object in the giant HII region NGC 3603," Astron. Astrophys. 167, L15-L16 (1986).

6. G. L. Rogers, "The process of image formation as the re-transformation of the partial coherence pattern of the object," Proc. Phys. Soc. 81, 323-331 (1963).

7. D. Korff, "Analysis of a method for obtaining near-diffractionlimited information in the presence of atmospheric turbulence," J. Opt: Soc. Am. 63, 971-980 (1973).

8. F. Roddier, "Triple correlation as a phase closure technique," Opt. Commun. 60, 145-149 (1986).

9. S. N. Karbelkar and R. Nityananda, "Atmospheric noise on the bispectrum in optical speckle interferometry," J. Astrophys. Astr. 8, 271-274 (1987).

10. A. C. S. Readhead, T. Nakajima, T. J. Pearson, G. Neugebauer, J. B. Oke, and W. L. W. Sargent, "Diffraction limited imaging with ground-based optical telescopes," Astron. J. 95, 1278-1296 (1988).

11. J. W. Goodman and J. F. Belsher, "Photon limited images and their restoration," Tech. Rep. RADC-TR-76-50 (Rome Air Development Center, New York, 1976).

12. J. W. Goodman and J. F. Belsher, "Precompensation and postcompensation of photon limited degraded images," Tech. Rep. RADC-TR-76-382 (Rome Air Development Center, New York, 1976).

13. J. W. Goodman and J. F. Belsher, "Photon limitations in imaging and image restoration," Tech. Rep. RADC-TR-77-175 (Rome Air Development Center, New York, 1977).

14. J. C. Dainty and A. H. Greenaway, "Estimation of spatial power spectra in speckle interferometry," J. Opt. Soc. Am. 69, 786-790 (1979).
15. B. Wirnitzer, "Bispectral analysis at low light levels and astronomical speckle masking," J. Opt. Soc. Am. A 2, 14-21 (1985).

16. A. N. Kolmogorov, "The local structure of turbulence in incompressible viscous fluid for very large Reynolds numbers," in Turbulence, Classical Papers on Statistical Theory, S. K. Friedlander and L. Topper, eds. (Wiley-Interscience, New York, 1961).

17. V. I. Tatarskii, Wave Propagation in a Turbulent Medium (McGraw-Hill, New York, 1961).

18. V. I. Tatarskii, The Effects on the Turbulent Atmosphere on Wave Propagation (Nauka, Moscow, 1967). (Translated by Israel Program for Scientific Translations, Keter, Jerusalem, 1971.)

19. J. Vernin, "Scidar measurements and model forecasting of free atmosphere turbulence," in Proceedings of the Second Workshop on ESO's Very Large Telescope, S. D'Odorico and J. P. Swings, eds. (European Southern Observatory, Garching, 1986), pp. 279-288.

20. C. Roddier and F. Roddier, "Interferometric seeing measurements at La Silla," in Proceedings of the Second Workshop on ESO's Very Large Telescope, S. D'Odorico and J. P. Swings, eds. (European Southern Observatory, Garching, 1986), pp. 269-278.

21. G. I. Taylor, "Statistical theory of turbulence," in Turbulence, Classical Papers on Statistical Theory, S. K. Friedlander and L. Topper, eds. (Wiley-Interscience, New York, 1961).

22. F. Roddier, "Atmospheric limitations to high angular resolution imaging," in Proceedings of the ESO Conference on The Scientific Importance of High Angular Resolution at Infrared and Optical Wavelengths, M. H. Ulrich and K. Kjar, eds. (European Southern Observatory, Garching, 1981), pp. 5-23.

23. J. W. Goodman, Statistical Optics (Wiley, New York, 1985).

24. J. E. Baldwin, C. A. Haniff, C. D. Mackay, and P. J. Warner, "Closure phase in high-resolution optical imaging," Nature 320, 595-597 (1986).

25. C. A. Haniff, C. D. Mackay, D. J. Titterington, D. Sivia, J. E. Baldwin, and P. J. Warner, "The first images from optical aperture synthesis," Nature 328, 694-696 (1987).

26. S. R. Kulkarni, California Institute of Technology, Pasadena, California 91126 (personal communication, 1987).

27. G. R. Ayers, M. J. Northcott, and J. C. Dainty, "Knox-Thompson and triple-correlation imaging through atmospheric turbulence," J. Opt. Soc. Am. A 5, 963-985 (1988). 